\title{
Wilson lines and gauge invariant off-shell amplitudes
}

\author{
Piotr Kotko \\ The H. Niewodniczański Institute of Nuclear Physics, Polish Academy of Sciences, \\ Radzikowskiego 152, 31-342 Kraków, Poland \\ E-mail: piotr.kotko@ifj.edu.pl
}

ABSTRACT: We study matrix elements of Fourier-transformed straight infinite Wilson lines as a way to calculate gauge invariant tree-level amplitudes with off-shell gluons. The offshell gluons are assigned "polarization vectors" which (in the Feynman gauge) are transverse to their off-shell momenta and define the direction of the corresponding Wilson line operators. The infinite Wilson lines are first regularized to prove the correctness of the method. We have implemented the method in a computer FORM program that can calculate gluonic matrix elements of Wilson line operators automatically. In addition we formulate the Feynman rules that are convenient in certain applications, e.g. proving the Ward identities. Using both the program and the Feynman rules we calculate a few examples, in particular the matrix elements corresponding to gauge invariant $g^{*} g^{*} g^{*} g$ and $g^{*} g^{*} g^{*} g^{*} g$ processes. An immediate application of the approach is in the high energy scattering, as in a special kinematic setup our results reduce to the form directly related to Lipatov's vertices. Thus the results we present can be directly transformed into Lipatov's vertices, in particular into $R R R P$ and $R R R R P$ vertices with arbitrary "orientation" of reggeized gluons. Since the formulation itself is not restricted to high-energy scattering, we also apply the method to a decomposition of an ordinary on-shell amplitude into a set of gauge invariant objects.

KEYwords: QCD Phenomenology

ArXiv EPRINT: 1403.4824 


\section{Contents}

1 Introduction 1

2 High energy amplitudes and gauge invariance 3

3 Basics of Wilson lines 5

$\begin{array}{lll}4 & \text { Formal developments } & 7\end{array}$

5 Automatic calculation of matrix elements 11

$6 \quad$ The Feynman rules 12

7 The Ward identities $\quad 14$

$\begin{array}{lll}7.1 & \text { Preliminaries } & 14\end{array}$

$\begin{array}{lll}7.2 & \text { One off-shell gluon } & 16\end{array}$

$\begin{array}{lll}7.3 & \text { Two off-shell gluons } & 17\end{array}$

8 Examples $\quad 19$

$\begin{array}{ll}\text { 8.1 Gauge invariant amplitude for } g^{*} g^{*} g & 19\end{array}$

8.2 Gauge invariant amplitude for $g^{*} g^{*} g^{*} g \quad 20$

$\begin{array}{lll}8.3 & \text { Gauge invariant amplitude for } g^{*} g^{*} g^{*} g^{*} g & 21\end{array}$

9 Gauge invariant decompositions $\quad 22$

10 Discussion and outlook $\quad 23$

A Direct calculation of gauge invariant $g^{*} g g$ matrix element 25

B The BRST invariance of the $\mathcal{R}$ operator $\quad 28$

\section{Introduction}

A basic off-shell object in QCD is a momentum space Green's function, i.e. the Fourier transform of a matrix element of time ordered field operators (in what follows we will consider only gluon operators for definiteness). In case of the most standard collinear factorization (see e.g. [1] for a review) the hard amplitude defining the perturbative core of the process is defined by the reduced connected Green's function, i.e. the external propagators are amputated, taken on-shell and contracted with polarization vectors. Thanks to on-shellness and transversality of the polarization vectors to corresponding momenta such amplitudes are gauge invariant. However, for processes occurring at high energies one 
often has to deal with so called high-energy $\left(\right.$ or $k_{T}$ ) factorization [2-5] (we put aside here the issues concerning the factorization breaking, see e.g. [6-10] for more details). In that case the reduction of the Green's function does not put all of the external legs on-shell; the remaining one or two off-shell legs are contracted with eikonal vectors corresponding to fast moving hadrons and off-shell momenta are restricted to be transverse to the pertinent eikonal vectors. There is however an issue related to gauge invariance of such objects. In general, in order to maintain the gauge invariance additional non-standard (i.e. not calculable from standard QCD Feynman rules) contributions are needed. One of the approaches is to use the Lipatov's effective action $[11,12]$ and interpret an off-shell gluon with additional contributions as an effective reggeized gluon $R$. Even at tree-level this approach is rather complicated for multiple final states. Therefore only recently some automatic methods to calculate such amplitudes for larger multiplicities have been developed [13-15] (examples of practical applications were presented in $[16,17]$ ). They use different methods than the Lipatov's effective action (see also e.g. [18] for yet another approach). In particular, the Lipatov's effective action uses Wilson lines, i.e. path ordered exponentials of color gauge fields, while the other methods do not refer to Wilson lines directly. However, the Wilson lines are viewed as the basic objects at very high energies (see e.g. [19] or the Color Glass Condensate formulation of QCD [20]), therefore they are always present in one or the other form. For instance in [13] they show up as eikonalized quarks. Actually, as we will see in the present paper also the additional contributions recovering the gauge invariance constructed in ref. [14] from the Slavnov-Taylor identities do correspond to a bremsstrahlung from a straight infinite Wilson line. Basing on this observation we will formulate a prescription to calculate off-shell gauge-invariant "amplitudes" by considering matrix elements of Fourier transforms of straight infinite Wilson line operators. The "momentum" of such an operator corresponds to an off-shell gluon (and additional contributions needed by the gauge invariance), while the direction of the Wilson line corresponds to its "polarization" vector. In our prescription the momenta and direction of a Wilson line are arbitrary, except that they have to be mutually transverse. This allows to apply the method also outside the high energy factorization approach as we will see.

In order to test the method we have implemented it in a computer program written in FORM [21]. It allows to calculate matrix elements of Wilson line operators for several external legs analytically. For instance, using the program a calculation of a process with four reggeized gluons with arbitrary "orientation" and an additional gluon emission, $R R R R g$, can be done automatically.

Let us collect at this point the main elements of the paper. i) Any tree-level amplitude with arbitrary number of gluonic off-shell legs and any number of on-shell legs, where the off-shell gluons have polarization vectors transverse to their off-shell momenta, can be made gauge invariant by assigning a proper infinite Wilson line operators to off-shell gluons. Those operators are at first sight ill-defined and we develop their regularized version to prove the correctness of the approach. To this end we also prove the Ward identities. ii) The off-shell amplitudes we consider here are more general than the ones appearing in the high-energy literature, but they reduce to the Lipatov's vertices with certain choice of the off-shell momenta and Wilson line directions (some of the contributions vanish with 
that choice). We check some explicit examples using the Feynman diagrams, in particular we give an example for the gauge invariant $g^{*} g^{*} g^{*} g$ process. iii) We construct a computer program that can calculate off-shell gluonic amplitudes automatically and analytically, using the presented method. Using the program we cross-check the result for $g^{*} g^{*} g^{*} g$ and calculate the gauge invariant $g^{*} g^{*} g^{*} g^{*} g$ matrix element.

The work is organized as follows. The first two sections are in a sense introductory. In section 2 we will introduce Wilson lines in the context of the off-shell amplitudes. We choose to do this by taking as an example the result of ref. [14] for off-shell high energy amplitudes. In section 3 we recall some basics concerning Wilson lines. Next, in section 4 we make some more formal definitions of the off-shell amplitudes using the Wilson lines. In section 5 we shortly present the computer program based on the method. We introduce the Feynman rules in section 6 and prove the Ward identities in section 7. In section 8 we give some examples of explicit calculations. Next, in section 9, we present a potentially interesting application of the present approach in decomposing ordinary amplitudes into gauge invariant pieces. Finally, we make some summarizing remarks in section 10.

\section{High energy amplitudes and gauge invariance}

In order to introduce the Wilson lines in the context of off-shell amplitudes, let us start with a short recollection of the high-energy factorization of Catani, Ciafaloni and Hautmann $(\mathrm{CCH})[3,5]$. For more detailed albeit compact review we refer e.g. to [14, 16]. In the original $\mathrm{CCH}$ approach a hadro- and lepto-production of heavy quarks was considered. At high energies, the relevant hard partonic sub-amplitudes turn out to be off-shell, i.e. we have to consider amplitudes $g^{*} g^{*} \rightarrow Q \bar{Q}$ or $\gamma g^{*} \rightarrow Q \bar{Q}$. They are defined by the Green's function with the on-shell legs amputated, while the off-shell gluon legs (including propagators) are contracted with so called eikonal vertices. To be more precise, if the momentum of the hadron $A$ is $p_{A}$ the corresponding eikonal vertex is just $p_{A}^{\mu}$ (modulo a prefactor). Moreover, the momentum $k_{A}$ of the corresponding off-shell leg has the form $x_{A} p_{A}+k_{T A}$, i.e. it is transverse to $p_{A}\left(k_{T} \cdot p_{A}=0, p_{A}^{2}=0\right)$. Since the CCH factorization is stated in the axial gauge, it turns out that the standard Feynman diagrams are enough to obtain the gauge invariant set of diagrams (recall we consider the heavy quark production case here). Moreover, for heavy quark lepto-production it is even true for any additional radiation of gluons. Therefore in [5] the $\mathrm{CCH}$ factorization was demonstrated to hold up to several loops for DIS heavy quark structure function. The last statement is also true for the so-called hybrid version of $\mathrm{CCH}$ factorization in hadron-hadron collision, i.e. where only one gluon is off-shell $[14,22]$. This approach is thought to be a good approximation to the full high-energy factorization in case of forward processes, e.g. forward jet production. However in the case of jets one usually needs also purely gluonic sub-processes. In that case the off-shell sub-process $g g^{*} \rightarrow g \ldots g$ is not gauge invariant. One can however still get the correct result with a particular choice of polarization vectors, but some modern methods relying on gauge invariance (e.g. the helicity method) cannot be used in that case. The most natural approach to the gauge invariance problem in the above context is just to see what kind of terms violate the gauge invariance and try to make use of that knowledge. 
This path was taken in ref. [14] and now we shall briefly recall this method and point out the connection to the Wilson line.

The high-energy gluonic amplitude $\mathcal{M}_{p_{A}}\left(\varepsilon_{1}, \ldots, \varepsilon_{N}\right)$ with a single off-shell leg with incoming momentum $k_{A}$ is defined by the following reduction formula

$$
\begin{aligned}
\mathcal{M}_{p_{A}}\left(\varepsilon_{1}, \ldots, \varepsilon_{N}\right)=\lim _{k_{A} \cdot p_{A} \rightarrow 0} \lim _{k_{1}^{2} \rightarrow 0} \ldots \lim _{k_{N}^{2} \rightarrow 0} & p_{A}^{\mu_{A}} k_{1}^{2} \varepsilon_{1}^{\mu_{1}} \ldots k_{N}^{2} \varepsilon_{N}^{\mu_{N}} \\
& \tilde{G}_{\mu_{A} \mu_{1} \ldots \mu_{N}}\left(k_{A}, k_{1}, \ldots, k_{N}\right),
\end{aligned}
$$

where $\varepsilon_{1}, \ldots, \varepsilon_{N}$ are polarization vectors of on-shell gluons with momenta $k_{1}, \ldots, k_{N}$ and $\tilde{G}$ is the momentum space Green's function. The internal (off-shell) propagators of $\tilde{G}$, including the leg with off-shell momentum $k_{A}$ are taken to be in the axial gauge with the gauge vector $p_{A}$, whereas the legs with momenta $k_{1}, \ldots, k_{N}$ are in the Feynman gauge. This is allowed as it is known that for the legs that are eventually on-shell one can choose a different gauge than for internal lines. Thanks to the first limit in (2.1) the momentum $k_{A}$ has the structure

$$
k_{A}^{\mu}=z_{A} p_{A}^{\mu}+k_{T A}^{\mu}
$$

with $k_{A}^{2}=-\left|\vec{k}_{T A}\right|^{2}$. The off-shell momentum $k_{A}$ has thus the structure complementary to the high-energy factorization described above. ${ }^{1}$ As already mentioned the amplitude $\mathcal{M}_{p_{A}}$ is in general not gauge invariant in the sense of the following Ward identity (unless we choose the polarization vectors in a special way)

$$
\mathcal{M}_{p_{A}}\left(\ldots, k_{i}, \ldots\right) \neq 0
$$

However, since we have the relation between the Green's function and $\mathcal{M}_{p_{A}}$ we can actually calculate the r.h.s. of (2.3) using the Slavnov-Taylor identities (for an elementary review of the Slavnov-Taylor identities see e.g. [23]). Furthermore, it turns out that within the gauge we are using the sum of all the gauge contributions with a proper treatment of external ghosts gives a "gauge-restoring amplitude" $\mathcal{W}$, such that $\tilde{\mathcal{M}}_{p_{A}}=\mathcal{M}_{p_{A}}+\mathcal{W}$ satisfies the Ward identity

$$
\tilde{\mathcal{M}}_{p_{A}}\left(\ldots, k_{i}, \ldots\right)=0
$$

In order to write the amplitude $\mathcal{W}$ in a compact manner, let us recall that any gluonic amplitude $\mathcal{M}$ may be decomposed into so called color-ordered amplitudes [24] as follows (we omit the polarization vectors here)

$$
\mathcal{M}=\sum_{\Pi^{\prime}\left(a_{1}, \ldots, a_{M}\right)} \operatorname{Tr}\left(t^{a_{1}} \ldots t^{a_{M}}\right) \mathcal{M}^{\left(a_{1} \ldots a_{M}\right)}
$$

where $a_{1}, \ldots, a_{M}$ are color indices of the gluons with momenta $k_{1}, \ldots, k_{M}$ respectively, $\Pi^{\prime}$ is the set of all non-cyclic permutations of the color indices, the matrices $t^{a}$ are generators of the $\mathrm{SU}(3)$ color group normalized as $\operatorname{Tr}\left(t^{a} t^{b}\right)=\delta^{a b} / 2$. The amplitude $\mathcal{M}^{\left(a_{1} \ldots a_{N}\right)}$

\footnotetext{
${ }^{1}$ There is a difference with respect to a definition of the corresponding amplitude given in [14], where additional factors were inserted to maintain collinear limit already at this stage. The relation is $\mathcal{M}=$ $\left|\vec{k}_{T A}\right| z_{A} \mathcal{M}_{p_{A}}$, where l.h.s. is the amplitude of [14].
} 


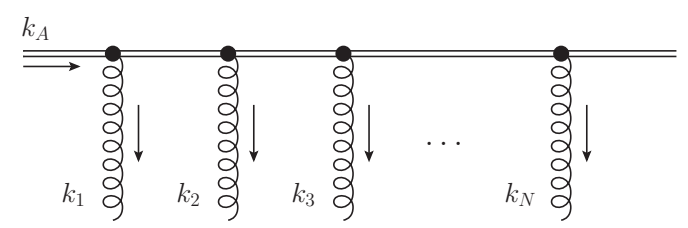

Figure 1. Graphical representation of the "gauge-restoring amplitude" $\mathcal{W}$. Each gluon is coupled via $p_{A}^{\mu}$ thus giving a factor $p_{A} \cdot \varepsilon_{i}$ for a gluon with momentum $k_{i}$ and polarization vector $\varepsilon_{i}$. The double-line propagator between subsequent emissions has the form $i / k \cdot p_{A}$ where $k$ is a momentum of the line. This picture will be further interpreted as a gauge link.

corresponds to a particular color ordering given in the superscript (see [25] for a review of color-ordering techniques). Specifically, in the present case the color decomposition for the off-shell amplitude reads

$$
\tilde{\mathcal{M}}_{p_{A}}=\sum_{\Pi^{\prime}\left(a_{A}, a_{1}, \ldots, a_{N}\right)} \operatorname{Tr}\left(t^{a_{A}} t^{a_{1}} \ldots t^{a_{N}}\right) \tilde{\mathcal{M}}_{p_{A}}^{\left(a_{A} a_{1} \ldots a_{N}\right)} .
$$

The result for the color-ordered version of the "gauge-restoring amplitude" $\mathcal{W}$ turns out to be very simple

$$
\begin{aligned}
\mathcal{W}^{\left(a_{A} a_{1} \ldots a_{N}\right)}\left(\varepsilon_{1}, \ldots, \varepsilon_{N}\right)= & -\left|\vec{k}_{T A}\right|\left(\frac{-g}{\sqrt{2}}\right)^{N-1} \varepsilon_{1} \cdot p_{A} \ldots \varepsilon_{N} \cdot p_{A} \\
& \frac{k_{1} \cdot p_{A}\left(k_{1}-k_{2}\right) \cdot p_{A} \ldots\left(k_{1}-k_{2}-\ldots-k_{N-1}\right) \cdot p_{A}}{} .
\end{aligned}
$$

The above result (2.7) has a very transparent structure and expresses certain bremsstrahlung contributions. Looking at the numerator, we see that each of the external on-shell gluons is coupled directly to the eikonal vector $p_{A}$. There are no triple or quartic gluon vertices as we have chosen the axial gauge with $p_{A}$ as the gauge vector; the propagator is thus always perpendicular to $p_{A}$ and all such couplings are eliminated. Next, looking at the denominator, we see that there are certain scalar propagators between each emission, i.e. we have $i / k \cdot p_{A}$ between each emission with $k$ being the momentum remaining after the last emission. This is illustrated in figure 1.

For people working with the collinear factorization the eikonal couplings and eikonal propagators are mainly familiar from the Feynman rules for PDFs $[1,26]$. They originate in a straight Wilson line connecting two fields separated on the light cone (and making the whole object gauge invariant). This may suggest, that the high-energy amplitude $\tilde{\mathcal{M}}_{p_{A}}$ is also related to a straight Wilson line. Indeed, as we shall see below the straight infinite Wilson line has a structure complementary with the structure of $\mathcal{W}$.

\section{Basics of Wilson lines}

Let us now recall some basic facts regarding gauge links (or Wilson lines; we shall use both terms interchangeably) and define our notation. For a more comprehensive review of Wilson lines in the context of Quantum Field Theory we refer e.g. to [23] (in that reference a gauge link is referred to as the "parallel-transporter" due to its geometrical interpretation). 
The Wilson line along the path $\mathcal{C}$ joining two space-time points $x, y$ is defined as

$$
[x, y]_{\mathcal{C}}=\mathcal{P} \exp \left\{i g \int_{C} d z_{\mu} A_{b}^{\mu}(z) t^{b}\right\}
$$

where $\mathcal{P}$ is the operation of ordering color matrices $t^{b}$ along the path. Its crucial property is the transformation law under the local gauge transformations $U(x)$

$$
[x, y]_{\mathcal{C}} \rightarrow U(x)[x, y]_{\mathcal{C}} U^{\dagger}(y)
$$

In many applications it is convenient to choose a straight line as the path $\mathcal{C}$. Defining the straight line to lie along the direction specified by the four vector $n$, it can be parametrized as

$$
z^{\mu}(s)=x^{\mu}+s n^{\mu}, \quad z^{\mu}(1)=y^{\mu} .
$$

Then, for a gauge link from $x$ to $y$ we have simply

$$
[x, y]_{n}=\mathcal{P} \exp \left\{i g \int_{0}^{1} d s n_{\mu} A_{b}^{\mu}(x+s n) t^{b}\right\}
$$

Note, that we have used a subscript $n$ to denote the path direction.

In the present paper we shall use the paths extending from minus to plus infinity. Let us introduce the following formal definition

$$
[x]_{n} \equiv \mathcal{P} \exp \left\{i g \int_{-\infty}^{\infty} d s n \cdot A_{b}(x+s n) t^{b}\right\}
$$

The subtleties concerning the (divergent) integration over $d s$ will be discussed below in section 4. Consider now the expansion of the gauge link defined above:

$$
\begin{aligned}
{[x]_{n}=\mathcal{P}\{} & 1+i g \int_{-\infty}^{\infty} d s n \cdot A_{b}(x+s n) t^{b} \\
& \left.+(i g)^{2} \frac{1}{2 !} \int_{-\infty}^{\infty} d s \int_{-\infty}^{\infty} d s^{\prime} n \cdot A_{b}(x+s n) n \cdot A_{b^{\prime}}\left(x+s^{\prime} n\right) t^{b} t^{b^{\prime}}+\ldots\right\}
\end{aligned}
$$

In order to utilize the path ordering we use the symmetry of the integrands with respect to $s, s^{\prime}, \ldots$ and obtain

$$
\begin{aligned}
{[x]_{n}=} & +i g \int_{-\infty}^{\infty} d s n \cdot A_{b}(x+s n) t^{b} \\
& +(i g)^{2} \int_{-\infty}^{\infty} d s \int_{-\infty}^{s} d s^{\prime} n \cdot A_{b}(x+s n) n \cdot A_{b^{\prime}}\left(x+s^{\prime} n\right) t^{b} t^{b^{\prime}}+\ldots
\end{aligned}
$$

Note, that the factorials originating from the expansion of the exponential cancel in the formula above. 


\section{Formal developments}

Let us now make a more formal connection of (2.7) to a gauge link. At this point we see from (3.7), that if the Wilson line direction is defined via the four vector $p_{A}$ appearing in (2.2), the external gluons will be contracted with $p_{A}$, as desired in view of eq. (2.7). The eikonal propagators in (2.7) will come out from the path ordered integrals. In the following we make those statements more formal.

First, note that the gauge link $[x]_{n}$ is gauge invariant on its own. This is due to eq. (3.2) and the fact that for a local gauge transformation $U(x)$ (more precisely for a small local gauge transformation) we have $U(x) \rightarrow 1$ for $|\vec{x}| \rightarrow \infty$. So $[x]_{n}$ is gauge invariant for any $x$ as long as $n$ has a nonzero spatial component. For definiteness we may restrict $n$ to be non-light-like at this point, but the gauge invariance of the matrix elements defined below will be maintained for any $n$.

Consider next a matrix element of an operator defined as follows

$$
\mathfrak{R}_{n}^{c}(k)=\int d^{4} x e^{i x \cdot k} \operatorname{Tr}\left\{\frac{1}{\pi g} t^{c}[x]_{n}\right\}
$$

More precisely, we consider

$$
\mathfrak{M}\left(n, \varepsilon_{1}, \ldots, \varepsilon_{N}\right) \stackrel{*}{=}\left\langle k_{1}, \varepsilon_{1}, c_{1} ; \ldots ; k_{N}, \varepsilon_{N}, c_{N}\left|\mathfrak{R}_{n}^{c}(k)\right| 0\right\rangle,
$$

where $\left|k_{i}, \varepsilon_{i}, c_{i}\right\rangle$ is an external on-shell gluon state in the Heisenberg picture with momentum $k_{i}$, polarization vector $\varepsilon_{i}$ and color $c_{i}$. The star adorning the equality sign means that only connected contributions (i.e. proportional to $\left.\delta^{4}\left(k_{A}+k_{1}+\ldots+k_{N}\right)\right)$ are to be taken into account. At this stage, the momentum $k$ is arbitrary, i.e. it is not restricted to the form similar to (2.2). However, we shall see below in section 6 that the form of the operator (4.1) assures that its matrix element is proportional to $\delta(k \cdot n)$ implying thus the high-energy kinematics (2.2) if $n=p_{A}$.

The matrix element (4.2) can be calculated explicitly in perturbation theory using field operators and the Wick theorem. The only complication is due to the integrals over the path parameters in (3.7) which are formally divergent. In order to define a suitable prescription, we propose the following finite-length (regularized) version of $[x]_{n}$

$$
[x]_{n}^{(\epsilon)} \equiv\left[x-\frac{2}{\epsilon} n, x+\frac{2}{\epsilon} n\right]
$$

with the path defined as

$$
z_{\epsilon}^{\mu}(s)=x^{\mu}+\frac{2}{\epsilon} \tanh \left(\frac{\epsilon s}{2}\right) n^{\mu}, \quad s \in(-\infty, \infty) .
$$

Note that

$$
z_{\epsilon}^{\mu}(s)=x^{\mu}+s n^{\mu}+\mathcal{O}\left(\epsilon^{2}\right)
$$


thus in the $\epsilon \rightarrow 0$ limit we recover $[x]_{n}$. Now, the regularized version of the expansion (3.7) reads

$$
\begin{aligned}
{[x]_{n}^{(\epsilon)}=} & 1+i g \int_{-\infty}^{\infty} d s \operatorname{sech}^{2} \lambda_{s} n \cdot A_{b}\left(z_{\epsilon}(s)\right) t^{b} \\
& +(i g)^{2} \int_{-\infty}^{\infty} d s \int_{-\infty}^{s} d s^{\prime} \operatorname{sech}^{2} \lambda_{s} \operatorname{sech}^{2} \lambda_{s^{\prime}} n \cdot A_{b}\left(z_{\epsilon}(s)\right) n \cdot A_{b^{\prime}}\left(z_{\epsilon}\left(s^{\prime}\right)\right) t^{b} t^{b^{\prime}}+\ldots
\end{aligned}
$$

where

$$
\lambda_{s}=\frac{\epsilon s}{2}
$$

Let us pass to the momentum space

$$
\begin{aligned}
{[x]_{n}^{(\epsilon)}=} & +i g \int \frac{d^{4} p}{(2 \pi)^{4}} e^{-i p \cdot x} n \cdot \tilde{A}_{b}(p) \int_{-\infty}^{\infty} d s \operatorname{sech}^{2} \lambda_{s} e^{-i \frac{2}{\epsilon} \tanh \lambda_{s} p \cdot n} t^{b} \\
& +(i g)^{2} \int \frac{d^{4} p}{(2 \pi)^{4}} \frac{d^{4} p^{\prime}}{(2 \pi)^{4}} e^{-i\left(p+p^{\prime}\right) \cdot x} n \cdot \tilde{A}_{b}(p) n \cdot \tilde{A}_{b^{\prime}}\left(p^{\prime}\right) \\
& \times \int_{-\infty}^{\infty} d s \int_{-\infty}^{s} d s^{\prime} \operatorname{sech}^{2} \lambda_{s} \operatorname{sech}^{2} \lambda_{s^{\prime}} e^{-i \frac{2}{\epsilon} \tanh \lambda_{s} p \cdot n} e^{-i \frac{2}{\epsilon} \tanh \lambda_{s^{\prime}} p^{\prime} \cdot n} t^{b} t^{b^{\prime}}+\ldots
\end{aligned}
$$

where $\tilde{A}$ is the Fourier-transformed gauge field. There are two types of integrals. The first one,

$$
I_{\epsilon}(p \cdot n)=\int_{-\infty}^{\infty} d s \operatorname{sech}^{2} \lambda_{s} e^{-i \frac{2}{\epsilon} \tanh \lambda_{s} p \cdot n}=\frac{2}{p \cdot n} \sin \left(\frac{2 p \cdot n}{\epsilon}\right) \equiv \delta_{\epsilon}(p \cdot n),
$$

is an approximation to the Dirac delta function,

$$
\lim _{\epsilon \rightarrow 0} \delta_{\epsilon}(x)=2 \pi \delta(x)
$$

since

$$
\int d x \delta_{\epsilon}(x)=2 \pi
$$

The second integral is

$$
J_{\epsilon}(s, p \cdot n)=\int_{-\infty}^{s} d s^{\prime} \operatorname{sech}^{2} \lambda_{s^{\prime}} e^{-i \frac{2}{\epsilon} \tanh \lambda_{s^{\prime}} p \cdot n}=\frac{i}{p \cdot n}\left(e^{-i \frac{2}{\epsilon} \tanh \lambda_{s} p \cdot n}-e^{i \frac{2}{\epsilon} p \cdot n}\right) .
$$

Those two types of integrals propagate for any of the terms in the expansion (4.8). The second exponent in the r.h.s. of (4.12) will not contribute to the result in the $\epsilon \rightarrow 0$ limit. This is because it oscillates rapidly and its contribution is zero due to the Riemann-Lebesgue lemma. Therefore for the regularized version of the operator (4.1) we obtain

$$
\begin{aligned}
\mathfrak{R}_{n}^{c(\epsilon)}(k)= & i n \cdot \tilde{A}_{b}(k) \frac{\delta_{\epsilon}(k \cdot n)}{2 \pi} 2 \operatorname{Tr}\left(t^{c} t^{b}\right) \\
& +i^{2} g \int \frac{d^{4} p}{(2 \pi)^{4}} n \cdot \tilde{A}_{b}(p) n \cdot \tilde{A}_{b^{\prime}}(k-p) \\
& \times \frac{i}{p \cdot n}\left[\frac{\delta_{\epsilon}(k \cdot n)}{2 \pi}+\mathcal{O}(\epsilon)\right] 2 \operatorname{Tr}\left(t^{c} t^{b} t^{b^{\prime}}\right)+\ldots,
\end{aligned}
$$


where the $\mathcal{O}(\epsilon)$ terms come from the second exponent in (4.12). In the limit $\epsilon \rightarrow 0$ we get

$$
\begin{aligned}
\Re_{n}^{c}(k)= & i n \cdot \tilde{A}_{b}(k) \delta(k \cdot n) 2 \operatorname{Tr}\left(t^{c} t^{b}\right) \\
& +i^{2} g \int \frac{d^{4} p}{(2 \pi)^{4}} \delta(k \cdot n) n \cdot \tilde{A}_{b}(p) n \cdot \tilde{A}_{b^{\prime}}(k-p) \frac{i}{p \cdot n} 2 \operatorname{Tr}\left(t^{c} t^{b} t^{b^{\prime}}\right)+\ldots
\end{aligned}
$$

From the above, we see that the operator $\mathfrak{R}_{n}(k)$ should be considered as a generalized function of $k \cdot n$. Therefore, instead of the regularization (4.3), (4.4) one can use the following more practical prescription known from the theory of the generalized functions; we use the infinite gauge links (3.7) with the following prescriptions for the path-ordered integrals

$$
\frac{i}{p \cdot n+i \epsilon}=e^{i s p \cdot n} \int_{-\infty}^{s} d s^{\prime} e^{-i s^{\prime} p \cdot n}
$$

and

$$
2 \pi \delta(p \cdot n)=\int_{-\infty}^{\infty} d s e^{-i s p \cdot n}
$$

Note, that the $i \epsilon$ prescription in (4.15) is the same as used in [1, 26] for collinear PDFs.

In the view of the above considerations, also the matrix element (4.2) should be considered as a generalized function of $k \cdot n$. It defines an object $\tilde{\mathcal{M}}_{n}$ we call off-shell gauge invariant amplitude for the process $g^{*}(k) g\left(k_{1}\right) \ldots g\left(k_{N}\right) \rightarrow 0$,

$$
\mathfrak{M}\left(n, \varepsilon_{1}, \ldots, \varepsilon_{N}\right)=\delta(k \cdot n) \delta^{4}\left(k_{A}+k_{1}+\ldots+k_{N}\right) \tilde{\mathcal{M}}_{n}\left(\varepsilon_{1}, \ldots, \varepsilon_{N}\right) .
$$

As a polarization vector for the off-shell gluon we understand a vector $\varepsilon$ defined as

$$
\varepsilon_{\mu}=n^{\nu} D_{\mu \nu}(k),
$$

where $D_{\mu \nu}(k)$ is the numerator of off-shell gluon propagator. In particular in the Feynman gauge we have simply

$$
\varepsilon^{\mu}=n^{\mu}
$$

and

$$
k \cdot \varepsilon=0
$$

If $n=p_{A}$, the amplitude $\tilde{\mathcal{M}}_{n}$ is the gauge invariant high-energy off-shell amplitude from section 2 .

We present a simple and instructive analytic calculation for a process $g^{*} g g$ in appendix A. It is of course very cumbersome to do a similar calculation by hand for larger multiplicities. However, the procedure is well defined and can be implemented in a computer program, provided it can deal with many terms (see section 5). Alternatively it is useful to construct the relevant Feynman rules. Besides the standard QCD rules, the rules related to an insertion of the operator (4.1) are needed. They will be constructed in section 6 . 
One can also define the matrix element of several gauge link operators, with which one can define the gauge invariant amplitude $\tilde{\mathcal{M}}_{n_{A} n_{B} n_{C} \ldots}$ with several off-shell gluons with momenta $k_{A}, k_{B}, k_{C}, \ldots$

$$
\begin{aligned}
\mathfrak{M}\left(n_{A}, n_{B}, n_{C} \ldots, \varepsilon_{1}, \ldots, \varepsilon_{N}\right)=\delta\left(k_{A} \cdot n_{A}\right) \delta\left(k_{B} \cdot n_{B}\right) \delta\left(k_{C} \cdot n_{C}\right) \ldots \\
\quad \delta^{4}\left(k_{A}+k_{B}+k_{C}+\ldots+k_{1}+\ldots+k_{N}\right) \tilde{\mathcal{M}}_{n_{A} n_{B} n_{C} \ldots}\left(\varepsilon_{1}, \ldots, \varepsilon_{N}\right) \\
\stackrel{*}{=}\left\langle k_{1}, \varepsilon_{1}, c_{1} ; \ldots ; k_{N}, \varepsilon_{N}, c_{N}\left|\Re_{n_{A}}^{c_{A}}\left(k_{A}\right) \mathfrak{R}_{n_{B}}^{c_{B}}\left(k_{B}\right) \Re_{n_{C}}^{c_{C}}\left(k_{C}\right) \ldots\right| 0\right\rangle
\end{aligned}
$$

by using the gauge links defined along $n_{A}, n_{B}, n_{C}, \ldots$ Let us note that although in the present paper we limit ourselves to gluonic on-shell states, the same prescription can be used for any other on-shell state.

There is a limitation for the allowed vectors $n_{X}, X=A, B, \ldots$ If some of $n_{X}$ are equal but not light-like, the parallel Wilson lines start to interact. This causes a problem. This is most easily seen when we realize that such interactions give rise to terms of the form

$$
\frac{i}{k_{X_{1}} \cdot n_{X_{2}}} n_{X_{1}} \cdot n_{X_{2}}, \quad X_{1}, X_{2}=A, B, C, \ldots
$$

which is divergent for $n_{X_{1}}=n_{X_{2}}$, unless $n_{X_{1}} \cdot n_{X_{2}}=0$ (recall that $k_{X_{i}} \cdot n_{X_{i}}=0, i=1,2$ ). Therefore, for definiteness, we assume that parallel Wilson lines have to be defined using null vectors.

The construction (4.21) can be used in the following way. Suppose we have a Green's function, which is reduced in such a way that some of the legs are on-shell and have standard polarization vectors, while some remain off-shell with momenta $k_{A}, k_{B}, \ldots$ and are contracted with certain "polarization vectors" $\varepsilon_{A}, \varepsilon_{B}, \ldots$ Call this reduced Green's function an off-shell amplitude $\mathcal{M}_{\varepsilon_{A} \varepsilon_{B} \ldots}$ - it is not gauge invariant. In order to find a gauge invariant extension $\tilde{\mathcal{M}}_{n_{A} n_{B} \ldots}$, we choose the vectors $n_{A}, n_{B}, \ldots$ satisfying

$$
k_{X} \cdot n_{X}=0, \varepsilon_{X \mu}=n_{X}^{\nu} D_{\mu \nu}\left(k_{X}\right), X=A, B, \ldots
$$

and use (4.21) with the gauge links directions along $n_{A}, n_{B}, \ldots$ In the Feynman gauge the gauge links directions correspond to polarization vectors of the off-shell gluons

$$
\varepsilon_{X}=n_{X}, X=A, B, \ldots
$$

The contribution to (4.21) coming from the first nontrivial term in expansion of the gauge links is precisely the starting non-gauge-invariant amplitude $\mathcal{M}_{n_{A} n_{B} \ldots}$ while the rest form an analog of $\mathcal{W}$ from section 2.

Since the delta functions $\delta\left(k_{X} \cdot n_{X}\right)$ in (4.21) come entirely from the insertion of $\mathfrak{R}_{n_{X}}^{c_{X}}\left(k_{X}\right)$ operators, one can define a more physical quantity by integrating the operators over the arguments of the deltas. To this end let us decompose any four vector to the component along $n_{X}$ as follows

$$
v^{\mu}=v^{\left(n_{X}\right)} n_{X}^{\mu}+v^{(n)} n^{\mu}+v_{T}^{\left(n_{X}, n\right) \mu}
$$


where $v_{T}^{\left(n_{X}, n\right)} \cdot n_{X}=v_{T}^{\left(n_{X}, n\right)} \cdot n=0$ and $n$ is an arbitrary four vector such that $n_{X} \cdot n \neq 0$. Using this we define

$$
\int d k_{X}^{\left(n_{X}\right)} \Re_{n_{X}}^{c_{X}}\left(k_{X}\right) \equiv \mathcal{R}_{n_{X}}^{c_{X}}\left(k_{X}^{(n)}, k_{T X}^{\left(n_{X}\right)}\right)
$$

Note, that the gauge link defining $\mathcal{R}$ does not depend on the $x$ component along $n$; the integration over $k_{X}^{\left(n_{X}\right)}$ gives the delta function $\delta\left(x^{\left(n_{X}\right)} n_{X}^{2}+x^{(n)} n_{X} \cdot n\right)$ (cf. the definition (4.1)) which in turn can be integrated over $x^{(n)}$ residing inside $d^{4} x$ measure in (4.1).

The action of the operator (4.26) on the vacuum state may be considered as a creation of a certain state corresponding to an off-shell gluon (in the terminology of [12] they would correspond to reggeized gluons if $n_{X}$ is eikonal momentum)

$$
\left\langle k_{X}, n_{X}, c_{X}\right|=\langle 0| \mathcal{R}_{n_{X}}^{c_{X}}\left(k_{X}^{(n)}, k_{T X}^{\left(n_{X}\right)}\right) .
$$

Such a state belongs to the cohomology of the Becchi-Rouet-Stora-Tyutin (BRST) transformation, similar to other "physical" asymptotic states as gluons or quarks. Although in principle it follows from the gauge invariance of infinite gauge links, we check this fact explicitly in appendix B, as it may not be clear that the prescription for path-ordered integrals preserves this property (see also section 7 ).

\section{$5 \quad$ Automatic calculation of matrix elements}

Having the definition (4.21) together with the prescriptions (4.15)-(4.16) the calculation of any tree level amplitude is a purely algebraic task. The only integrals to be performed are of exponential nature, which in turn can also be done symbolically. Therefore, we have constructed a program written in FORM [21], that calculates (4.21) automatically. It does not refer to the Feynman rules, instead it uses the Wick theorem (see an example calculation presented in the appendix A). Therefore in the future fermionic and other gauge fields can be added relatively easy. Although FORM is very powerful and can deal with huge expressions, the number of terms that appear due to the Wick theorem is often enormous. Therefore it was a crucial task to find a reasonable algorithm to deal with the Wick contractions. At the moment the program was tested on amplitudes with time ordered product of at most 13 gauge fields on a standard laptop. This corresponds for example to a matrix element of four Wilson line operators and a gluon. Another difficulty to overcome was related to a simplification of expressions; it is necessary to use momentum conservation and relations between invariants at the intermediate steps of calculation and the program does it automatically. The color algebra is also done automatically, so that the results are given in the color-ordered representation [24, 25].

The program is called OGIME - an alias for Off-shell Gauge Invariant Matrix Elements. It is available from the author's web pages [27] or via email upon request. The technical details concerning the program are beyond the scope of this paper and will be presented elsewhere. 


\section{The Feynman rules}

In order to construct the relevant Feynman rules for an insertion of $\Re_{n_{X}}^{c_{X}}\left(k_{X}\right)$ operators in the QCD matrix element, let us again consider one of the terms in its expansion, say the $m$-th term

$$
\begin{aligned}
& \frac{1}{2 \pi g}(i g)^{m} \int d^{4} x e^{-i k_{X} \cdot x} \int_{-\infty}^{\infty} d s_{1} \int_{-\infty}^{s_{1}} d s_{2} \ldots \int_{-\infty}^{s_{m-1}} d s_{m} \\
& \quad n_{X} \cdot A_{b_{1}}\left(x+s_{1} n_{X}\right) \ldots n_{X} \cdot A_{b_{m}}\left(x+s_{m} n_{X}\right) 2 \operatorname{Tr}\left(t^{c_{X}} t^{b_{1}} \ldots t^{b_{m}}\right) \\
& =\int \frac{d^{4} p_{1}}{(2 \pi)^{4}} \ldots \frac{d^{4} p_{m}}{(2 \pi)^{4}} \delta^{4}\left(p_{1}+\ldots+p_{m}+k_{X}\right) \int_{-\infty}^{\infty} \frac{d s_{1}}{\pi g} \ldots \int_{-\infty}^{s_{m-1}} d s_{m} \\
& \quad \delta_{B_{m} A_{0}} t_{A_{0} B_{0}}^{c_{X}} e^{-i s_{1} p_{1} \cdot n_{X}} \delta_{B_{1} A_{2}} e^{-i s_{2} p_{2} \cdot n_{X}} \ldots \delta_{B_{m-1} A_{m}} e^{-i s_{m} p_{m} \cdot n_{X}} \\
& \quad i g t_{A_{1} B_{1}}^{b_{1} n_{X}} \cdot \tilde{A}_{b_{1}}\left(p_{1}\right) \ldots i g t_{A_{m} B_{m}}^{b_{m}} n_{X} \cdot \tilde{A}_{b_{m}}\left(p_{m}\right),
\end{aligned}
$$

where $\tilde{A}$ are the Fourier-transformed gluon fields and the capital letter indices $A_{i}, B_{i}$ are the fundamental color indices (summed over). The above structure can be interpreted as follows. The fields are separated on a line, graphically represented by a double line carrying color quantum numbers in fundamental representation and the total momentum $k_{X}$ which we assign to its beginning. Each gluon field with the color $b$ is attached to this line via $i g t_{A B}^{b} n_{X}^{\mu}$ vertex. The field attachments are separated by eikonal propagators which follow from the integrals along the path:

$$
\begin{aligned}
\int_{-\infty}^{\infty} \frac{d s_{1}}{\pi g} \ldots \int_{-\infty}^{s_{m-1}} d s_{m} t_{A_{0} B_{0}}^{c_{X}} \delta_{B_{m} A_{0}} e^{-i s_{1} p_{1} \cdot n_{X}} \delta_{B_{1} A_{2}} e^{-i s_{2} p_{2} \cdot n_{X}} \ldots \delta_{B_{m-1} A_{m}} e^{-i s_{m} p_{m} \cdot n_{X}} \\
=\int_{-\infty}^{\infty} \frac{d s_{1}}{\pi g} \ldots \int_{-\infty}^{s_{m-2}} d s_{m-1} \delta_{B_{m} A_{0}} t_{A_{0} B_{0}}^{c_{X}} \\
\quad e^{-i s_{1} p_{1} \cdot n_{X}} \ldots \delta_{B_{m-2} A_{m-1}} e^{-i s_{m-1}\left(p_{m-1}+p_{m}\right) \cdot n_{X}} \frac{i \delta_{B_{m-1} A_{m}}}{p_{m} \cdot n_{X}+i \epsilon} \\
=\frac{2 \delta_{B_{m} A_{0}} t_{A_{0} B_{0}}^{c_{X}}}{g} \delta\left(p_{1} \cdot n_{X}+\ldots p_{m} \cdot n_{X}\right) \frac{i \delta_{B_{1} A_{2}}}{\left(p_{1}+\ldots+p_{m}\right) \cdot n_{X}+i \epsilon} \ldots \frac{i \delta_{B_{m-1} A_{m}}}{p_{m} \cdot n_{X}+i \epsilon} .
\end{aligned}
$$

Above, we have used $i \epsilon$ prescription (4.15) as explained in the previous section. In the last line we encounter the delta function (4.16), which together with momentum conservation will give the delta function $\delta\left(k_{X} \cdot n_{X}\right)$. The factor 2 in front of the color delta function will assure the correct matching with the standard QCD result when the on-shell limit is taken (of course after multiplying by inverse propagators for $k_{X}$ momenta).

In figure 2 we have gathered the resulting Feynman rules for insertion of (4.1) into a QCD matrix element. The top diagram is a "skeleton" for a gauge link and originates in the delta function in (6.1) with the prefactor. Its left-most side has the momentum and adjoint color quantum number of the $\mathfrak{R}_{n_{X}}^{c_{X}}\left(k_{X}\right)$ operator. The momentum (and the color) flows to the right. The end of the gauge link (the right-most part) has momentum zero. Between the beginning and the end of the Wilson line gluons can be attached, according to the rest of the rules given in figure 2 .

In order to better trace the color (and momentum) flow in a diagram (especially when more gauge links are present) it is convenient to "bend" the gauge link as shown in figure 3. 


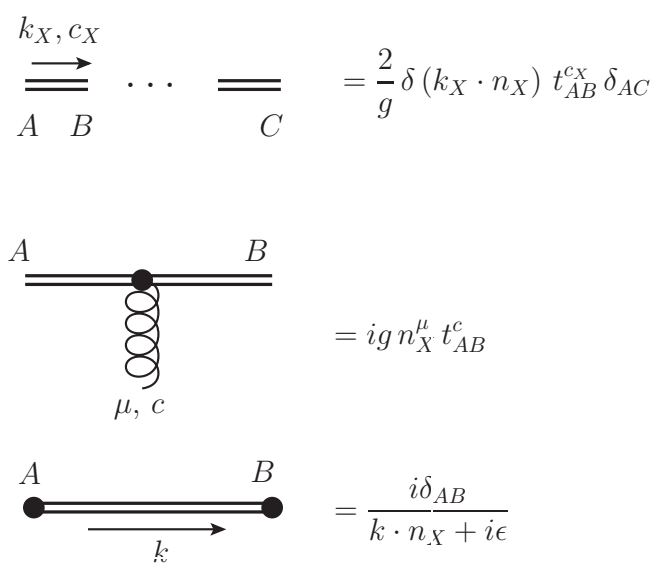

Figure 2. The Feynman rules for an insertion of the operator $\mathfrak{R}_{n_{X}}^{c_{X}}\left(k_{X}\right)$. The top diagram is a "skeleton" for a gauge link; it consist in the transversality constraint, the color projection, and color delta function eventually giving a trace. The right-most end of the double line has zero momentum. The middle diagram represents the coupling of a gluon to the gauge link. The bottom diagram represents the gauge-link propagator originating in path-ordered integrals.

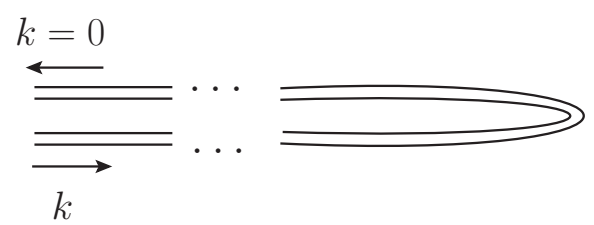

Figure 3. The improved graphical representation for the Feynman rule for the gauge link (the dots stand for gluon attachments and gauge-link propagators). Such a representation is more convenient when more gauge links are present and is easy to convert to color-ordered rule.

In case of two off-shell gluons, one can alternatively draw the two gauge links as the topmost and the most bottom features, remembering that the direction of a momentum flow (and the trace direction) has to be reversed for the bottom-most gauge link.

In the following it will be convenient to work with color-ordered amplitudes defined in eq. (2.5). The standard color-ordered QCD Feynman rules are listed e.g. in the appendix of [25] or [14]. We have to supply this list by the rules for gauge links. This is straightforward, as gauge link contribution gives always a color trace, similar to a gluon sub-amplitude (recall that gluon vertices give products of traces due to $\left.f_{a b c}=-2 i\left(\operatorname{Tr}\left(t^{a} t^{b} t^{c}\right)-\operatorname{Tr}\left(t^{c} t^{b} t^{a}\right)\right)\right)$. We omit color indices, drop all the color matrices and assign $1 / \sqrt{2}$ for each coupling of a gluon to a gauge link. Further, we consider only planar diagrams with the "bent" gauge link (of course we can "unroll" it keeping track of the color and momentum flow). Note, however that our definition (2.5) assumes the standard normalization of the color generators $\operatorname{Tr}\left(t^{a} t^{b}\right)=\delta^{a b} / 2$, while the usual color-ordered Feynman rules do assume that $\operatorname{Tr}\left(t^{a} t^{b}\right)=\delta^{a b}$ (see e.g. [25]). In order to compensate for this mismatch one has to multiply the result by a factor $(\sqrt{2})^{N}$, where $N$ is the number of external on-shell momenta. 


\section{The Ward identities}

We have seen in the beginning of the section 4 that the gauge links defining the operator (4.1) are manifestly gauge invariant with respect to small gauge transformations. However, the path integrals residing inside the expansion are divergent and a regularization is required (or a prescription to give a physical meaning to the integrals). The regularization leads to finite-length Wilson lines, thus violating the gauge invariance argument. After the limit $\epsilon \rightarrow 0$ is taken the gauge invariance should be recovered. Therefore it is necessary and instructive to prove that the off-shell amplitudes defined via (4.21) together with the prescription to define the path integrals (4.15) do satisfy the Ward identities. Moreover, this exercise nicely illustrates the reason the method of ref. [14] (and recalled in section 2) does actually work. We consider only gluonic amplitudes hereafter; the amplitudes with quarks can be analyzed even more easily in a similar manner. For another study of the Ward identities in the context of Lipatov's effective action see [28].

\subsection{Preliminaries}

Before we face our main task, we will gather some preliminary results that will be useful later. Let us start with the Slavnov-Taylor identity for partially reduced Green's function. Suppose we have the Green's function (in the Feynman gauge) and we contract one of the external legs with the corresponding momentum. The Slavnov-Taylor identity states that it equals to the sum of contributions with ghosts, diagrammatically

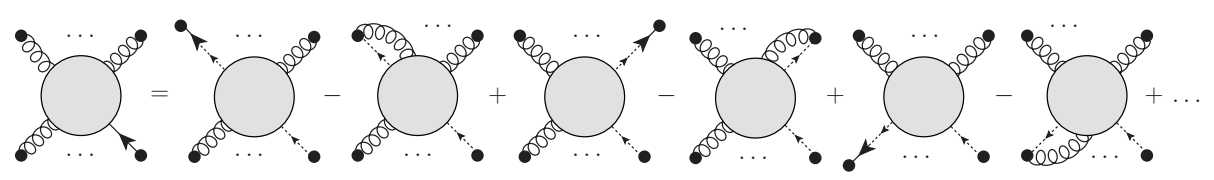

The large arrows denote the contraction with the momentum of a line, the big dots indicate that a line has a propagator, whereas the horizontal dots between the upper and bottom legs remind that there are several legs of the same type. A dot with a ghost and a gluon connected to it is the the vertex due to the BRST transformation, namely $g f^{a b d} A_{\mu}^{b} c^{d}$ with $c^{d}$ being a ghost field. Finally, the ellipses after the plus sign denote similar diagrams for the remaining legs. Suppose now that we want to reduce the Green's function in such a way that the bottom legs are on-shell. The standard reduction formula is applied to those lines, i.e. they are multiplied by the inverse propagators and contracted with the polarization vectors. After such reduction, we are left only with the diagrams where the outgoing ghosts are off-shell (they exit in the upper part of the diagrams from (7.1)).

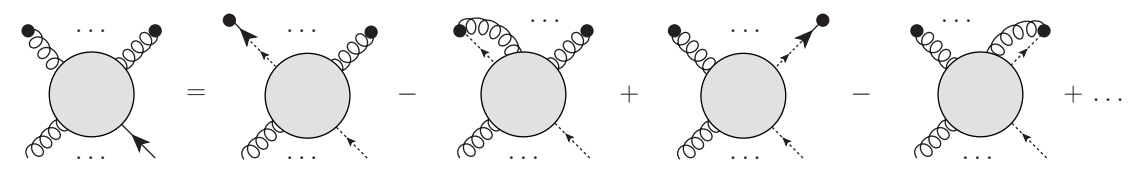

Above we have indicated that the bottom legs are on-shell (the big dots are missing there because the propagators were amputated) while the top are still off-shell.

In what follows, in order to simplify the diagrammatic analysis we shall work with color ordered amplitudes. Moreover, for further reduction of the number of diagrams we shall 
work in the axial gauge for internal propagators with the gauge vector $n^{\prime}$, in general not light-like. For that choice of gauge the identity (7.2) can still be applied (cf. [14]); in the axial gauge ghosts couple to gluons via $g f^{a b c} n^{\prime \mu}$ and the ghost propagator is $i /\left(k \cdot n^{\prime}+i \epsilon\right)$.

Consider now an attachment of a sub-amplitude with $N$ external on-shell gluons to the gauge link defined via vector $n, n \neq n^{\prime}$. When we replace one of the polarization vectors by the corresponding momentum of the line we get

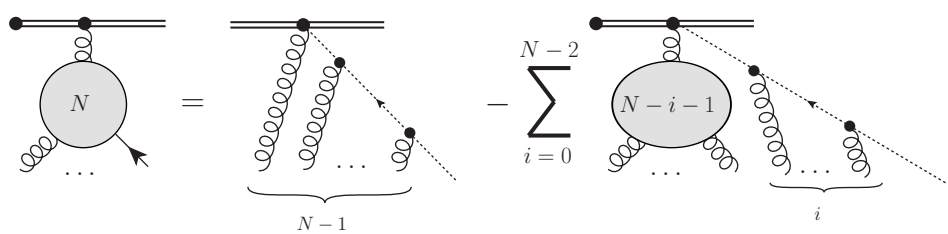

where the numbers on the blobs denote the number of external on-shell legs. This identity follows directly from the Slavnov-Taylor identity (7.2) in the axial gauge and the transversality of the axial-gauge gluon propagator to the gluon-ghost coupling, i.e.

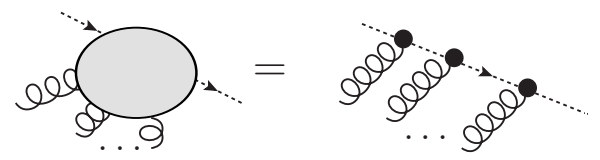

Note, that the first term on the r.h.s. of (7.3) has no gauge-link propagator as it was canceled by the outgoing momentum of the ghost contracted with $n$

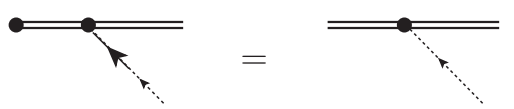

The color-ordered rule for the BRST gluon-ghosts coupling is taken with the plus sign if the ghost is to the right of the gluon (an exchange leads to a minus sign). We use here the "unbent" gauge link with momentum flowing from the left to the right.

In order to derive the Ward identity for a gauge link, consider now the set of all the contributions attached to a gauge link, starting from a certain point (i.e. we cut the gauge link at some point and include the gauge-link propagator)

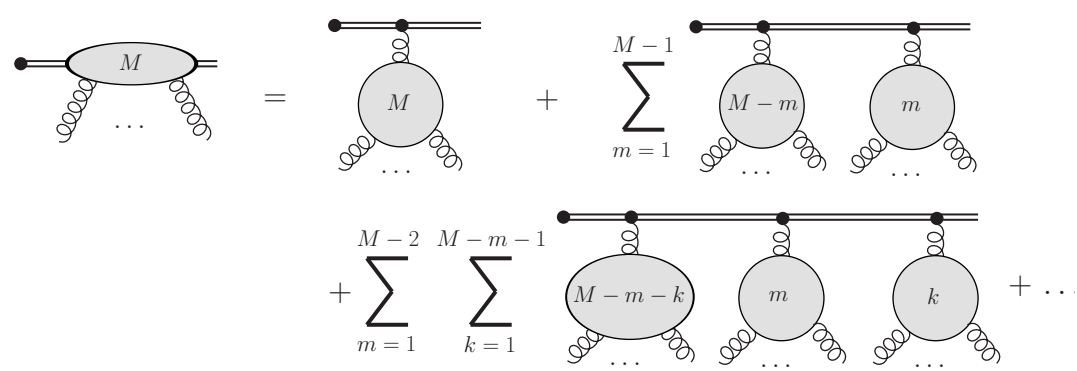

where the ellipses after the plus sign denote the contributions with more blobs attached to the gauge link. Let us now replace the polarization vector by the momentum for the rightmost gluon. Since for two consecutive blobs we have (following (7.3)) 

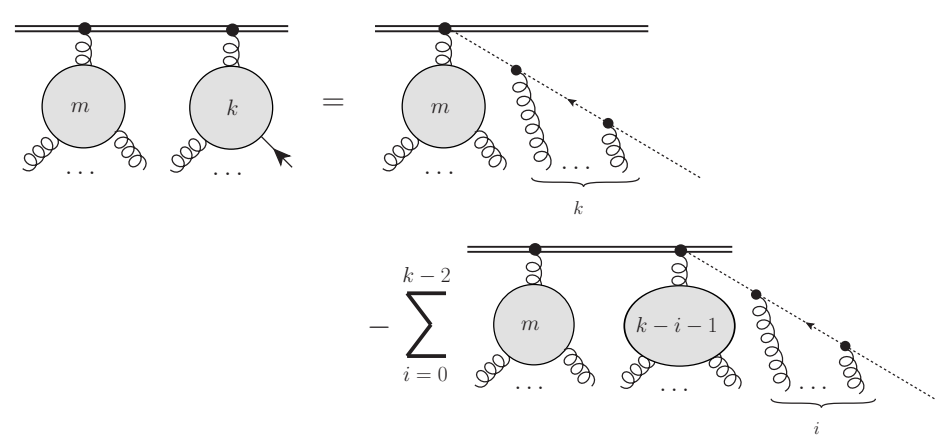

we find that the Ward identity for the whole series of the blobs attached to the gauge link takes the following simple form

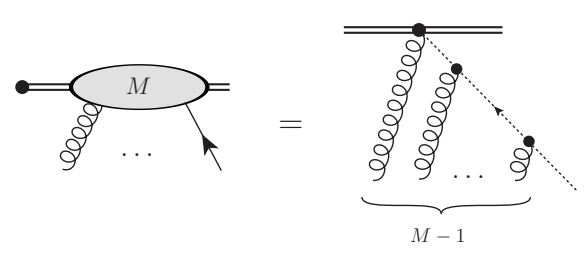

All the terms canceled between the consecutive blobs in (7.6), except the first term.

Similar identities can be derived (with somewhat more effort) for the replacements of the other gluons then the rightmost. Let us now discuss the Ward identities for (4.21) for one and two Wilson line operator insertions.

\subsection{One off-shell gluon}

Since for a single off-shell gluon we have one Wilson line operator, the Ward identity follows directly from (7.8). Let us suppose the gauge link is defined with the momentum $k_{A}$ and the direction $n_{A}$. We have

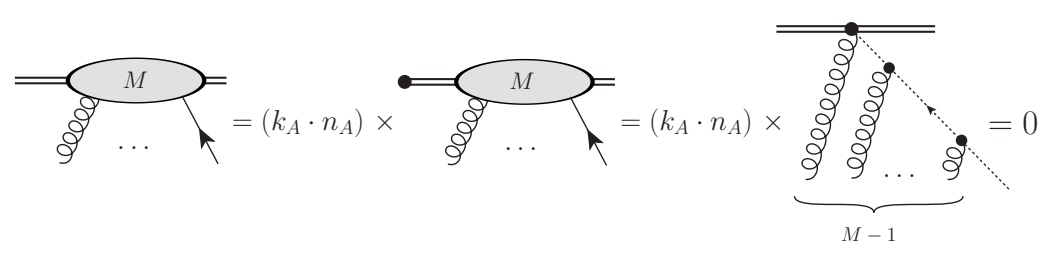

To obtain the first equality we have multiplied and divided by the gauge link propagator. The second equality follows directly from (7.8). Finally, the last equality follows in the distributional sense, i.e. after integration over $k^{\left(n_{A}\right)}$ due to the delta $\delta\left(k_{A} \cdot n_{A}\right)$ which resides in the gauge link.

Let us note, that when the axial gauge vector $n^{\prime}=n_{A}$, the only contributions to (7.2) are precisely of the form of the rightmost term of (7.9). This leads to the conclusion, that in such a case the gauge link can be mimicked by the sum of the Slavnov-Taylor gauge terms, with the external ghosts traded to longitudinal gluons projected on $n_{A}$. This is precisely what has been used in ref. [14]. 


\subsection{Two off-shell gluons}

Now let us consider the situation with two Wilson line operators. Let us choose the momentum and the direction of the first gauge link to be $k_{A}$ and $n_{A}$, while the for the second we choose $k_{B}$ and $n_{B}$. The relevant matrix element is graphically expressed as

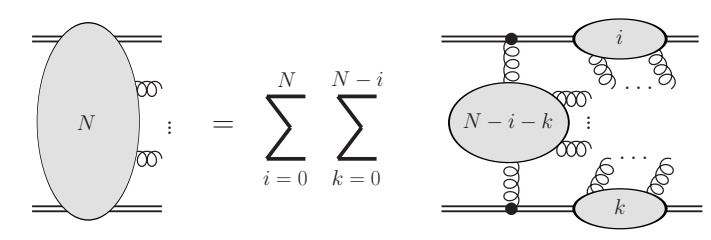

Since we use here the "unbent" Feynman rule for the gauge links (see the penultimate paragraph of section 6), one has to remember that for the bottom gauge link the momentum flows from the right to the left (the opposite to the top one). Let us now replace the polarization vector of the top on-shell gluon by its momentum. It is convenient to split (7.10) into three distinct topologies, i.e. we consider

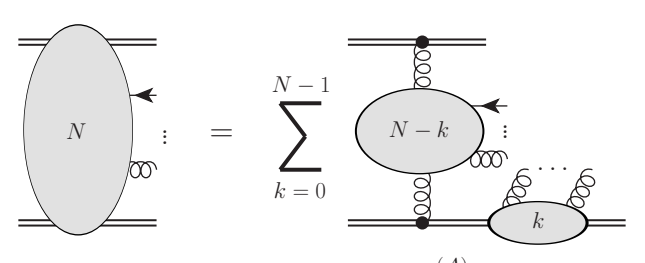

(A)

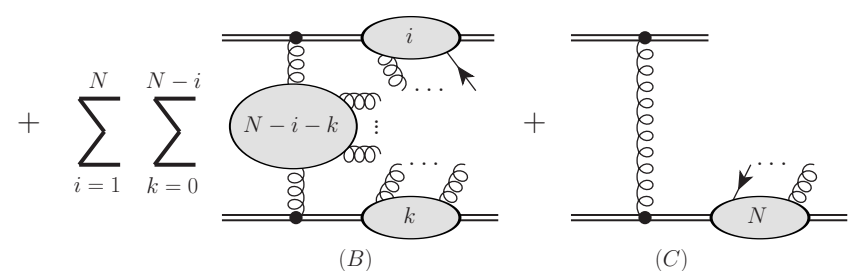

Consider now the $(A)$ and $(B)$ terms. Using $(7.8),(7.2)$ and the fact that $k_{A} \cdot n_{A}=0$ due to the delta function $\delta\left(k_{A} \cdot n_{A}\right)$ residing in the upper gauge link, they become

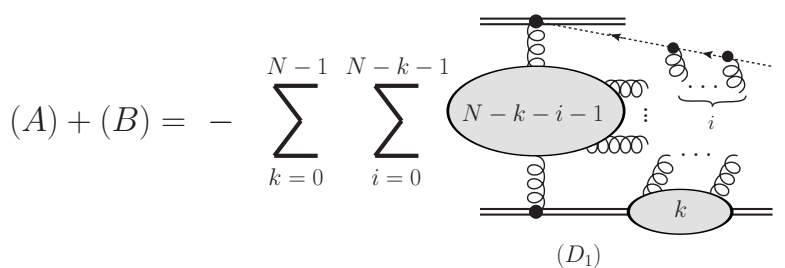

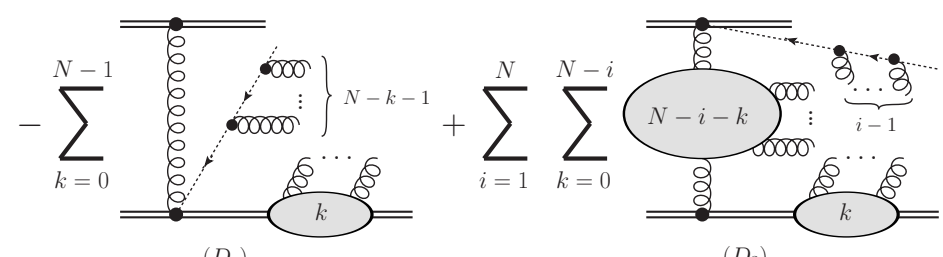

Note that the first and the last sum cancel against each other

$$
\left(D_{1}\right)+\left(D_{3}\right)=0
$$


so we are left with the middle sum $\left(D_{2}\right)$. We have to show that it cancels with the term $(C)$ of (7.11). The last reads



In order to proceed, let us note the following Slavnov-Taylor-like identity for a gauge link; it follows simply from the momentum conservation, the form of the gauge-link propagator and the asymmetry of the color-ordered BRST gluon-ghost vertex

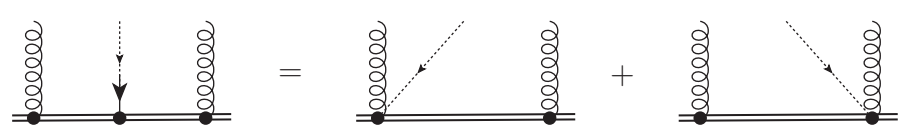

Expanding the blob with $k$ legs in the diagram $\left(C_{3}\right)$ according to (7.6) and using the above result we have

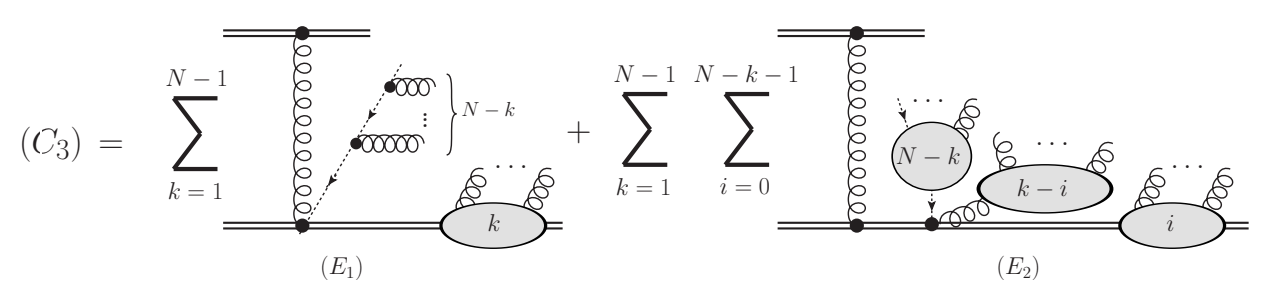

After reshuffling the sums, the second term $\left(E_{2}\right)$ cancels with $\left(C_{4}\right)$ due to (7.4). Finally, it is easy to see that

$$
\left(E_{1}\right)+\left(C_{1}\right)+\left(D_{2}\right)=0 .
$$

We have shown that all the terms on the r.h.s. of (7.11) cancel against each other and thus the Ward identity is indeed fulfilled. Similar proofs for more gauge links are also possible, but very cumbersome to carry out explicitly. For three and four gauge links we have checked the Ward identities using explicit results presented in section 8 . 

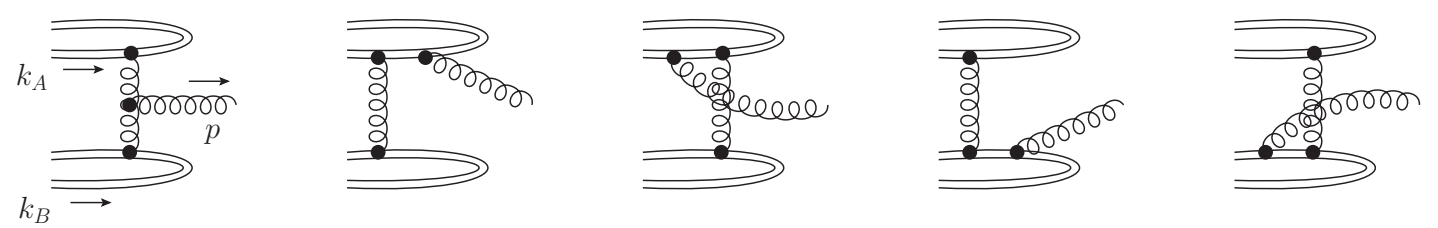

Figure 4. The diagrams for the gauge invariant process $g^{*}\left(k_{A}\right) g^{*}\left(k_{B}\right) \rightarrow g(p)$. The momentum flow is displayed for the first diagram only.

Let us note that the proofs are in a sense purely algebraic and they do not refer to the form of the off-shell momenta or gauge link directions, the only property that is relevant here is their mutual transversality.

\section{Examples}

\subsection{Gauge invariant amplitude for $g^{*} g^{*} g$}

Let us start with a simple example of a gauge invariant matrix element for the following process

$$
g^{*}\left(k_{A}\right) g^{*}\left(k_{B}\right) \rightarrow g(p) .
$$

The momentum conservation is $k_{A}+k_{B}=p$ with $p^{2}=0$. We choose the Feynman gauge and polarization vectors to be $\varepsilon_{A}, \varepsilon_{B}, \varepsilon$ satisfying $\varepsilon_{A} \cdot k_{A}=0, \varepsilon_{B} \cdot k_{B}=0, \varepsilon \cdot p=0$. Let the colors of the gluons be $c_{A}, c_{B}$ and $c$ respectively.

The relevant Feynman diagrams are displayed in figure 4. Using our Feynman rules we get

$$
\begin{aligned}
\tilde{\mathcal{M}}_{\varepsilon_{A} \varepsilon_{B}}(\varepsilon)= & \varepsilon_{A}^{\mu} \varepsilon_{B}^{\nu} \varepsilon^{\gamma} \frac{1}{k_{A}^{2} k_{B}^{2}} f^{c_{A} c_{B} c} V_{\mu \beta \gamma}\left(k_{A}, k_{B},-p\right) \\
& -2 i g \frac{\varepsilon_{A} \cdot \varepsilon_{B} \varepsilon \cdot \varepsilon_{A}}{k_{B}^{2} p \cdot \varepsilon_{A}}\left[\operatorname{Tr}\left(t^{c_{A}} t^{c_{B}} t^{c}\right)-\operatorname{Tr}\left(t^{c_{A}} t^{c} t^{c_{B}}\right)\right] \\
& -2 i g \frac{\varepsilon_{A} \cdot \varepsilon_{B} \varepsilon \cdot \varepsilon_{B}}{k_{A}^{2} p \cdot \varepsilon_{B}}\left[\operatorname{Tr}\left(t^{c_{B}} t^{c_{A}} t^{c}\right)-\operatorname{Tr}\left(t^{c_{B}} t^{c} t^{c_{A}}\right)\right] \\
= & i g f^{c_{A} c_{B} c}\left\{\frac{-i \varepsilon_{A}^{\mu} \varepsilon_{B}^{\nu} \varepsilon^{\gamma} V_{\mu \beta \gamma}\left(k_{A}, k_{B},-p\right)}{k_{A}^{2} k_{B}^{2}}+\right. \\
& \left.\frac{\varepsilon_{A} \cdot \varepsilon_{B} \varepsilon \cdot \varepsilon_{A}}{k_{B}^{2} p \cdot \varepsilon_{A}}-\frac{\varepsilon_{A} \cdot \varepsilon_{B} \varepsilon \cdot \varepsilon_{B}}{k_{A}^{2} p \cdot \varepsilon_{B}}\right\}
\end{aligned}
$$

where $V_{\alpha \beta \gamma}^{a b c}\left(k_{1}, k_{2}, k_{3}\right)=f^{a b c} V_{\alpha \beta \gamma}\left(k_{1}, k_{2}, k_{3}\right)$ with

$$
V_{\alpha \beta \gamma}\left(k_{1}, k_{2}, k_{3}\right)=-g\left[\eta_{\alpha \beta}\left(k_{1}-k_{2}\right)_{\gamma}+\eta_{\beta \gamma}\left(k_{2}-k_{3}\right)_{\alpha}+\eta_{\gamma \alpha}\left(k_{3}-k_{1}\right)_{\beta}\right]
$$

is the three-gluon coupling. It is easy to see that the above result recovers the Lipatov's RRP vertex [12], provided $\varepsilon_{A}$ and $\varepsilon_{B}$ are replaced by $n_{-}$and $n_{+}$where $n_{ \pm}=(1,0,0, \mp 1)$ define the "plus" and "minus" light-cone directions. Actually, to get the precise equality, 


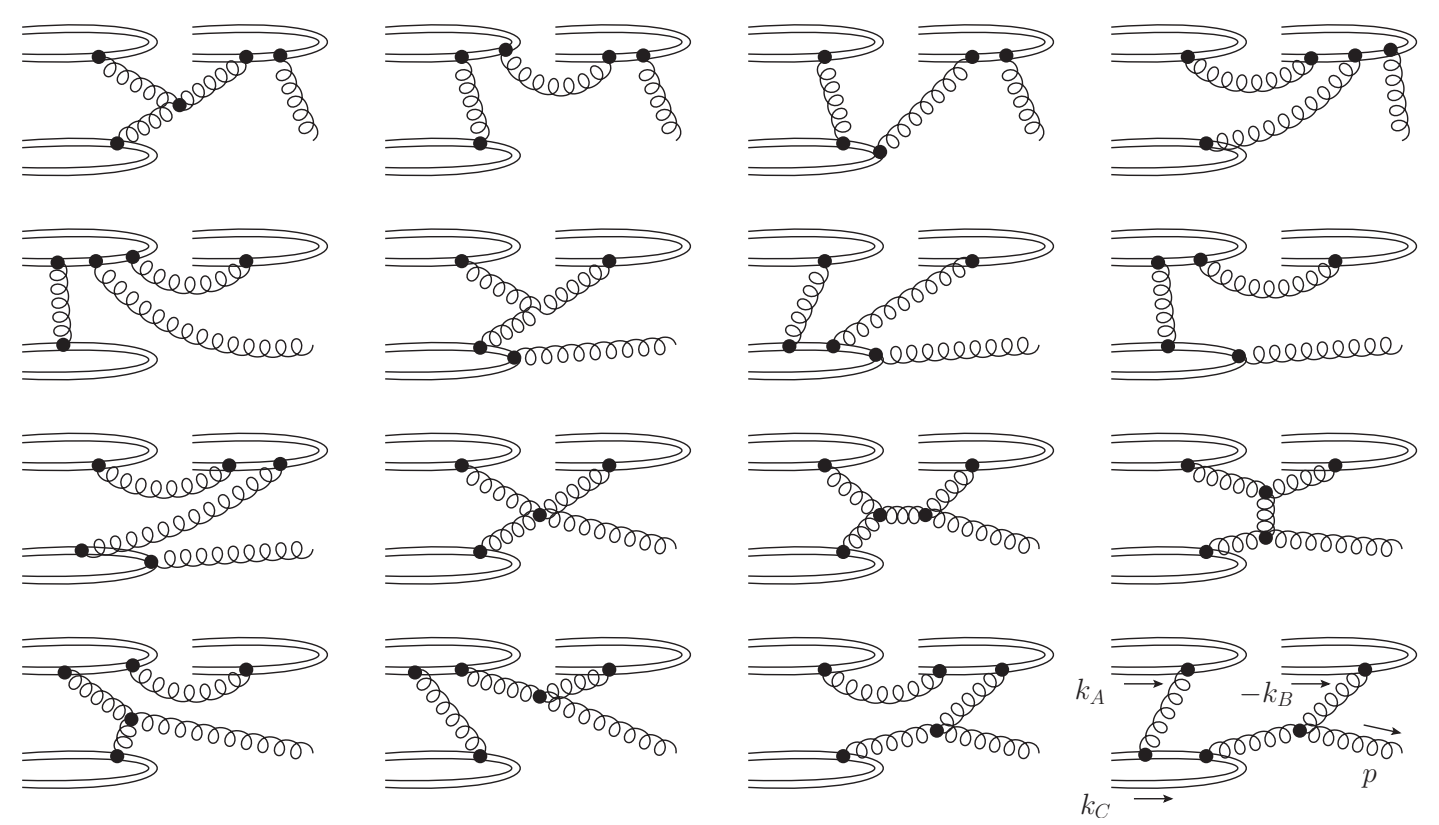

Figure 5. The color-ordered diagrams for the process $g^{*}\left(k_{A}\right) g^{*}\left(k_{C}\right) \rightarrow g^{*}\left(k_{B}\right) g(p)$ and color order $\left(c_{A}, c_{B}, c, c_{C}\right)$. The momentum flow is displayed in the last diagram only.

our eq. (8.6) has to be multiplied by the inverse propagators of the off-shell gluons, i.e. $k_{A}^{2} k_{B}^{2}$. We will see in the next subsection, that such one-to-one correspondence between our matrix elements and the Lipatov vertices does not hold for more off-shell gluons.

There are two color ordered amplitudes $\tilde{\mathcal{M}}_{\varepsilon_{A} \varepsilon_{B}}^{\left(c_{A} c_{B}\right)}$ and $\tilde{\mathcal{M}}_{\varepsilon_{A} \varepsilon_{B}}^{\left(c_{B} c_{A} c\right)}$ that contribute to eq. (8.6). They are easy to read out - they are the coefficients of the color traces. We have checked that they match the result obtained automatically form the OGIME program discussed in section 5 .

\subsection{Gauge invariant amplitude for $g^{*} g^{*} g^{*} g$}

We turn now to the gauge-invariant amplitude for the process with three off-shell gluons and one on-shell. Let us assign the momenta as follows

$$
g^{*}\left(k_{A}\right) g^{*}\left(k_{C}\right) \rightarrow g^{*}\left(k_{B}\right) g(p)
$$

and assign the color quantum numbers to be $c_{A}, c_{C}, c_{B}, c$ respectively. The momentum conservation reads $k_{A}+k_{C}=k_{B}+p$ with $p^{2}=0$. Let us assume, that the on-shell gluon has the polarization vector $\varepsilon$ whereas the off-shell gluons are assigned the polarization vectors $\varepsilon_{A}, \varepsilon_{B}, \varepsilon_{C}$ transverse to the momenta $k_{A}, k_{B}, k_{C}$ accordingly.

Let us work in the Feynman gauge and consider the color-ordered amplitude with the color order $\left(c_{A}, c_{B}, c, c_{C}\right)$. There are 16 color ordered diagrams shown in figure 5 . It is straightforward to calculate them using the color ordered Feynman rules given in section 6 (and the ones for standard QCD listed e.g. in [14]). The relevant gauge links are defined using $\varepsilon_{A}, \varepsilon_{B}, \varepsilon_{C}$ vectors. 
The result from the Feynman diagrams has been cross-checked with the OGIME program. We append the analytic result to the paper in a text file RRRG.txt (online resource 1). The result is given in the most general form, when $\varepsilon_{A} \neq \varepsilon_{B} \neq \varepsilon_{C}$. If any of those vectors coincide they should be light-like (see section 4). We have checked explicitly that our result satisfies the Ward identity

$$
\mathcal{M}_{\varepsilon_{A} \varepsilon_{B} \varepsilon_{C}}^{\left(c_{A}, c_{B}, c, c_{C}\right)}(p)=0 .
$$

The other color ordered amplitudes can be obtained by suitable exchanges of the pairs $\left(k_{X}, \varepsilon_{X}\right), X=A, B, C$. The full result for the squared amplitude is just given by the sum of color ordered amplitudes squared (let us recall that it is not a general rule and holds for at most five external legs, see e.g. [25]).

Let us stress that the above result corresponds to the known result for RRRG vertex given in [29] only for a special case, such that $\varepsilon_{A}=n_{+}, \varepsilon_{B}=\varepsilon_{C}=n_{-}$and $k_{A}=$ $x_{A} n_{-}+k_{A T}, k_{B}=x_{B} n_{+}+k_{B T}, k_{C}=x_{C} n_{+}+k_{C T}$ with $n_{ \pm}$defined in the previous section. With the above choice of the kinematics the diagrams with a gluon joining the Wilson lines with momenta $k_{B}$ and $k_{C}$ vanish. The precise relation to the result of [29] is the following: our result should be multiplied by $i k_{A}^{2} k_{B}^{2} k_{C}^{2} / 6$. The origin of inverse propagators is obvious, while the factor of 6 comes entirely from our convention of defining the $\mathfrak{R}$ operator (there are three of them, each contributes a factor of 2). Namely, our definition is such that when the on-shell limit is taken (after multiplying by inverse offshell propagators), we obtain the standard text-book result. This is also the case for the Lipatov vertices listed in [12], but not for [29]. This is evident if we compare the RRP vertices presented in both papers - they differ by a factor of 4 .

We underline, that our result is more general than the one listed in [29], as it allows for arbitrary "orientation" of reggeized gluons. The special cases can be obtained by imposing additional restrictions on the $\varepsilon_{A}, \varepsilon_{B}, \varepsilon_{C}$ vectors.

\subsection{Gauge invariant amplitude for $g^{*} g^{*} g^{*} g^{*} g$}

Finally, let us turn to the process

$$
g^{*}\left(k_{A}\right) g^{*}\left(k_{D}\right) \rightarrow g^{*}\left(k_{B}\right) g^{*}\left(k_{C}\right) g(p) .
$$

The polarization vectors are respectively $\varepsilon_{A}, \varepsilon_{D}, \varepsilon_{B}, \varepsilon_{C}, \varepsilon$ and the colors $c_{A}, c_{D}, c_{B}, c_{C}, c$.

The calculation using the Feynman diagrams is rather lengthy even for color-ordered amplitude, therefore we have calculated this amplitude using OGIME program only. The result is appended to the paper in a text file RRRRG.txt (online resource 2) for the most general form, when $\varepsilon_{A} \neq \varepsilon_{B} \neq \varepsilon_{C} \neq \varepsilon_{D}$. If any of those vectors coincide they should be lightlike (see section 4 ). We have explicitly checked that the result satisfies the Ward identity.

We note, that for certain choice of polarization vectors $\varepsilon_{A}=n_{+}, \varepsilon_{B}=n_{-}, \varepsilon_{C}=n_{-}$, $\varepsilon_{D}=n_{-}$(where $n_{ \pm}$were defined in the previous section) the result corresponds to $R R R R P$ Lipatov's vertex, calculated in [30]. Similar as previously, the result we present is however more general as it allows for any "orientation" for reggeized gluons. 


\section{Gauge invariant decompositions}

Let us now discuss another interesting application of gauge invariant off-shell matrix elements. As we have indicated it already a few times, they can be built using almost arbitrary gauge link directions. Below, we will use this fact to decompose an ordinary gauge invariant amplitude into gauge invariant off-shell sub-amplitudes.

Consider first a standard tree-level off-shell current $J^{\mu}\left(\varepsilon_{1}, \ldots, \varepsilon_{N} ; k\right)$ in the Feynman gauge, where $\varepsilon_{1}, \ldots, \varepsilon_{N}$ are polarization vectors of $N$ on-shell gluons with momenta $k_{1}, \ldots, k_{N}$ respectively and $k=k_{1}+\ldots+k_{N}$ (we omit color indices for brevity). Let us note that we may write

$$
J^{\mu}\left(\varepsilon_{1}, \ldots, \varepsilon_{N} ; k\right)=\sum_{i=0}^{2} J_{\nu}\left(\varepsilon_{1}, \ldots, \varepsilon_{N} ; k\right) \epsilon_{i}^{\nu}(k) \epsilon_{i}^{\mu}(k) d_{i}(k) .
$$

The auxiliary (in general complex) four-vectors $\epsilon_{i}(k)$ are defined in such a way that

$$
\begin{aligned}
k \cdot \epsilon_{i}(k)=0, \quad \epsilon_{i}(k) \cdot \epsilon_{j}(k) & =d_{i}(k) \delta_{i j}, \\
\sum_{i=0}^{2} \epsilon_{i}^{\nu}(k) \epsilon_{i}^{\mu}(k) d_{i}(k) & =g^{\mu \nu}-\frac{k^{\mu} k^{\nu}}{k^{2}},
\end{aligned}
$$

where

$$
d_{0}(k)=\left\{\begin{array}{ll}
1 & k^{2}<0 \\
-1 & k^{2}>0
\end{array}, \quad d_{1}(k)=d_{2}(k)=-1 .\right.
$$

The identity (9.1) follows because of the current conservation $J^{\mu}\left(\varepsilon_{1}, \ldots, \varepsilon_{N} ; k\right) k_{\mu}=0$. The auxiliary four vectors $\epsilon_{i}(k)$ can be easily constructed for any off-shell four momentum $k$. For instance, if $k$ is space-like we go to a frame where $k=\left(0,0,0, \sqrt{\left|k^{2}\right|}\right)$ and define $\epsilon_{0}(k)=(1,0,0,0), \epsilon_{1}(k)=(0, \sin \phi, \cos \phi, 0), \epsilon_{2}(k)=(0,-\cos \phi, \sin \phi, 0)$ and eventually go back to the original frame. Obviously, the four-vectors $\epsilon_{i}(k)$ are not unique.

The off-shell current $J^{\mu}\left(\varepsilon_{1}, \ldots, \varepsilon_{N} ; k\right)$ is not gauge invariant. Thanks to the fact that $\epsilon_{i}(k) \cdot k=0$ we may however define a gauge invariant off-shell current as follows

$$
\begin{aligned}
\tilde{J}_{i}^{\mu}\left(\varepsilon_{1}, \ldots, \varepsilon_{N} ; k\right) & =\tilde{J}_{i}\left(\varepsilon_{1}, \ldots, \varepsilon_{N} ; k\right) \epsilon_{i}^{\mu}(k) d_{i}(k) \\
& =\left[-\frac{1}{k^{2}} J_{\nu}\left(\varepsilon_{1}, \ldots, \varepsilon_{N} ; k\right) \epsilon_{i}^{\nu}(k)+G_{i}\left(\varepsilon_{1}, \ldots, \varepsilon_{N} ; k\right)\right] \epsilon_{i}^{\mu}(k) d_{i}(k),
\end{aligned}
$$

where

$$
\tilde{J}_{i}\left(\varepsilon_{1}, \ldots, \varepsilon_{N} ; k\right) \stackrel{*}{=}\left\langle k_{1}, \varepsilon_{1} ; \ldots ; k_{N}, \varepsilon_{N}\left|\mathcal{R}_{\epsilon_{i}}(k)\right| 0\right\rangle
$$

and $G_{i}\left(\varepsilon_{1}, \ldots, \varepsilon_{N} ; k\right)$ are contributions coming from additional emissions from the Wilson line residing in $\mathcal{R}_{\epsilon_{i}}(k)$ (they are generalization of the "gauge-restoring amplitude" $\mathcal{W}$ from section 2). The above equation leads to the following decomposition of the standard offshell current into gauge invariant and gauge non-invariant pieces

$$
J^{\mu}\left(\varepsilon_{1}, \ldots, \varepsilon_{N} ; k\right)=-\sum_{i=0}^{2}\left[k^{2} \tilde{J}_{i}^{\mu}\left(\varepsilon_{1}, \ldots, \varepsilon_{N} ; k\right)-G_{i}\left(\varepsilon_{1}, \ldots, \varepsilon_{N} ; k\right) \epsilon_{i}^{\mu}(k) d_{i}(k)\right] .
$$


Consider now a particular example, namely a color-ordered on-shell amplitude for the four gluon process $g g g g \rightarrow 0$. It can be expressed in terms of color-ordered currents as follows

$$
\begin{aligned}
& \mathcal{M}^{(1,2,3,4)}\left(\varepsilon_{1}, \varepsilon_{2}, \varepsilon_{3}, \varepsilon_{4}\right)=J_{\mu}^{(1,2)}\left(\varepsilon_{1}, \varepsilon_{2} ; k_{12}\right) \frac{i}{k_{12}^{2}} J^{\mu(3,4)}\left(\varepsilon_{3}, \varepsilon_{4} ; k_{34}\right) \\
& +J_{\mu}^{(4,1)}\left(\varepsilon_{1}, \varepsilon_{4} ; k_{14}\right) \frac{i}{k_{14}^{2}} J^{\mu(2,3)}\left(\varepsilon_{2}, \varepsilon_{3} ; k_{23}\right)+V_{4}^{(1,2,3,4)}\left(\varepsilon_{1}, \varepsilon_{2}, \varepsilon_{3}, \varepsilon_{4}\right)
\end{aligned}
$$

where

$$
V_{4}^{(1,2,3,4)}\left(\varepsilon_{1}, \varepsilon_{2}, \varepsilon_{3}, \varepsilon_{4}\right)=\frac{g^{2}}{2} V_{4}^{\alpha_{1} \alpha_{2} \alpha_{3} \alpha_{4}} \varepsilon_{1 \alpha_{1}} \varepsilon_{2} \alpha_{2} \varepsilon_{3 \alpha_{3}} \varepsilon_{4 \alpha_{4}}
$$

is the color-ordered four gluon coupling. The color-ordered objects, with the color order indicated in the superscript parentheses were defined in section 2. In what follows, we drop the arguments of color-ordered objects; such a shortcut notation does not lead to any confusion here. Using the gauge invariant currents (9.5) we may write

$$
\mathcal{M}^{(1,2,3,4)}=i\left(k_{12}^{2} \tilde{J}^{(1,2)} \cdot \tilde{J}^{(3,4)}+k_{14}^{2} \tilde{J}^{(4,1)} \cdot \tilde{J}^{(2,3)}+\tilde{V}_{4}^{(1,2,3,4)}\right),
$$

where the gauge invariant (color-ordered) four-gluon vertex is defined as

$$
\begin{aligned}
\tilde{V}_{4}^{(1,2,3,4)}= & -i V_{4}^{(1,2,3,4)}-G^{(1,2)} \cdot \tilde{J}^{(3,4)}-\tilde{J}^{(1,2)} \cdot G^{(3,4)}+\frac{1}{k_{12}} G^{(1,2)} \cdot G^{(3,4)} \\
& -G^{(4,1)} \cdot \tilde{J}^{(2,3)}-\tilde{J}^{(4,1)} \cdot G^{(2,3)}+\frac{1}{k_{14}} G^{(4,1)} \cdot G^{(2,3)}
\end{aligned}
$$

The scalar product in the expressions above is defined as

$$
A \cdot B=\sum_{i=0}^{2} A_{i} B_{i} d_{i} .
$$

Let us summarize what we have done. We have used the matrix elements of Wilson lines to decompose a gauge invariant on-shell amplitude (here we have used a simple example of four-leg amplitude) to manifestly gauge invariant objects. Such decomposition is however not unique, as the gauge invariant objects depend on the choice of four-vectors $\epsilon_{i}$. Similar decompositions are possible also for more complicated objects.

The practical applications of such decompositions are under study. At this point, as an example, let us turn the attention to the ref. [31], where a decomposition of an on-shell amplitude to $g g g^{*}$ subprocess (and the reminder) was used in order to study the spin effects in the QCD evolution. There, however, the gauge-violating part was abandoned as it was a non-leading-logarithmic contribution.

\section{Discussion and outlook}

We start by summarizing the main points of the paper. The notion "off-shell amplitude" is motivated by the high-energy factorization and refers to an ordinary scattering amplitude, where some of the gluonic legs are not taken on-shell, plus contributions needed to 
maintain the gauge invariance. We have defined such off-shell amplitudes in eq. (4.21) via matrix elements of the Fourier transforms of straight infinite Wilson line operators. The "polarization vectors" of off-shell gluons correspond (in the Feynman gauge) to the directions of the Wilson lines. We have implemented the method in the FORM program, called OGIME, and tested it for certain processes involving several off-shell and on-shell gluons. In the present work only tree-level gluonic matrix elements were studied.

As there are many applications of Wilson lines in the high-energy literature, let us briefly compare their various instances to the present one. Let us start with the Lipatov's effective action $[11,12]$. The Lipatov's vertices are formulated in terms of two auxiliary fields $A_{+}$and $A_{-}$. Due to the equations of motion they are related to the Wilson lines defined in terms of two light-like four vectors $n_{+}, n_{-}$defining the "plus" and "minus" light cone components. The Wilson lines are however not infinite there, but they start at certain fixed space-time position and span to infinity. If we defined the matrix elements in terms of the $\mathcal{R}$ operators with the Wilson lines defined using only $n_{ \pm}$four vectors we would recover the Lipatov's vertices. It was also demonstrated explicitly in section 8. In [19, 32] and [33] the Wilson line operators are infinite, but are explicitly taken to have "plus" or "minus" light-cone component set to zero. This corresponds to our integrated $\mathfrak{R}$ operators, cf. eq. (4.26), with gauge link direction again defined to be along the "plus" or "minus" light-cone vector. The present approach is more general - the gauge link directions need not to be constrained to the "plus" or "minus" light-cone vectors and there are additional contributions related to one gluon exchanges between the Wilson lines.

Let us also compare the present approach to the one of ref. [13]. There, also a set of eikonal Feynman rules was introduced, using however completely different method. Again, for our $\mathfrak{R}$ operators taken to be along the hadrons momenta the results from both methods coincide. However, at the moment the method [13] takes into account at most two off-shell gluons within high energy kinematics described in section 2 .

One of the main purposes of this work was to provide a practical formulation for offshell amplitudes. We may claim that we have reach that goal, as the relevant program, the Feynman rules, as well as explicit calculations were presented. Since the program calculates the amplitudes analytically, it obviously has certain limitations; already for five external legs with arbitrary polarization vectors the results are rather lengthy. Therefore, as far as the future applications for analytic calculations are concerned it is rather more interesting to include other Standard Model fields in the program. This is planned for the near future.

\section{Acknowledgments}

The author is grateful for many useful discussions and reading the manuscript to A. van Hameren and K. Kutak. Useful comments and questions were also given by L. Motyka, M. Sadzikowski and W. Schafer, to whom the author is grateful as well.

The work was partially supported by the Polish National Science Center grants LIDER/02/35/L-2/10/NCBiR/2011 and DEC-2011/01/B/ST2/03643.

The Feynman diagrams were drawn using JaxoDraw program [34]. 


\section{A Direct calculation of gauge invariant $g^{*} g g$ matrix element}

In order to illustrate how the OGIME program calculates the matrix elements let us present a sample calculation. It uses only very basics of the Quantum Field Theory.

The precise definition of the process is as follows

$$
g^{*}\left(k_{A}\right) g\left(k_{1}\right) \rightarrow g\left(k_{2}\right)
$$

where the gluons have colors $c_{A}, a_{1}, a_{2}$ and polarizations $\varepsilon_{A}, \varepsilon_{1}^{\lambda_{1}}, \varepsilon_{2}^{\lambda_{2}}$ respectively. Here, unlike in the main text we explicitly indicate the polarization projections $\lambda_{1}, \lambda_{2}$.

For the purpose of the explicit derivations let us define the necessary field operators in the Heisenberg picture. We have for a gluon field

$$
A_{\mu}^{c}(x)=\int \widetilde{d q} \frac{1}{\sqrt{2 E_{q}}} \sum_{\lambda}\left[\hat{a}_{c}^{\lambda}(q) \varepsilon_{\mu}^{\lambda}(q) e^{-i q \cdot x}+\hat{a}_{c}^{\lambda \dagger}(q) \varepsilon_{\mu}^{\lambda *}(q) e^{i q \cdot x}\right]
$$

where $E_{q}=\sqrt{q^{2}+\vec{q}^{2}}$ and the hatted quantities are creation/annihilation operators with the commutation relations

$$
\left[\hat{a}_{c}^{\lambda}(q), \hat{a}_{c^{\prime}}^{\lambda^{\prime} \dagger}\left(q^{\prime}\right)\right]=(2 \pi)^{3} \delta^{3}\left(\vec{q}-\vec{q}^{\prime}\right) \delta_{\lambda \lambda^{\prime}} \delta_{c c^{\prime}}
$$

We introduce the following shorthand notation for the integration measures

$$
\frac{d^{4} p}{(2 \pi)^{4}} \equiv \widehat{d p}, \quad \frac{d^{3} p}{(2 \pi)^{3}} \equiv \widetilde{d p}
$$

A one-particle on-shell state with momentum $q$, color $c$ and polarization vector $\varepsilon^{\lambda}(q)$ is defined as

$$
|q, \lambda, c\rangle \equiv\left|q, \varepsilon^{\lambda}(q), c\right\rangle=\sqrt{2 E_{q}} \hat{a}_{c}^{\lambda \dagger}(q)|0\rangle .
$$

The relevant interaction part of the Yang-Mills action is (skipping ghost and gaugefixing parts)

$$
S_{I}=\int d^{4} x\left(g V_{g g g}(x)+g^{2} V_{g g g g}(x)\right)
$$

where

$$
\begin{aligned}
V_{g g g}(x) & =f_{a b c} \partial_{\mu} A_{\nu}^{a}(x) A^{b \mu}(x) A^{c \nu}(x), \\
V_{g g g g}(x) & =-\frac{1}{4}\left(f_{a b c} A_{\mu}^{b}(x) A_{\nu}^{c}(x)\right)^{2},
\end{aligned}
$$

For further purposes, let us write $V_{g g g}$ interactions in the form

$$
V_{g g g}(x)=f_{a b c} \int \widehat{d p} \int d^{4} y(-i) p_{\mu} e^{-i(x-y) \cdot p} A_{\nu}^{a}(y) A^{b \mu}(x) A^{c \nu}(x) .
$$

The matrix element we are to calculate is

$$
\mathfrak{M}\left(\varepsilon_{A} ; \varepsilon_{1}, \varepsilon_{2}\right) \stackrel{*}{=} \int d^{4} x e^{i x \cdot k_{A}}\left\langle k_{1}, \lambda_{1}, a_{1}\left|\operatorname{Tr}\left\{\frac{1}{\pi g} t^{c_{A}}[x]_{\varepsilon_{A}}\right\}\right| k_{2}, \lambda_{2}, a_{2}\right\rangle .
$$


First, we use the Gell-Mann-Low formula what accounts in inserting the exponential of the Yang-Mills interaction (A.6), $\exp \left(-i S_{I}\right)$, switching to the interaction picture (in what follows we shall not indicate this explicitly) and time ordering the fields. Taking the connected diagrams only we are left with

$$
\mathfrak{M}\left(\varepsilon_{A} ; \varepsilon_{1}, \varepsilon_{2}\right)=\int d^{4} x e^{i x \cdot k_{A}}\left(M_{1}+M_{2}\right)=\tilde{M}_{1}+\tilde{M}_{2},
$$

where the tildes denote the Fourier transforms with respect to $x$ and

$$
\begin{aligned}
M_{1}= & -\frac{i^{2} g}{\pi} \operatorname{Tr}\left(t^{c_{A}} t^{b}\right) \int_{-\infty}^{\infty} d s \int d^{4} y\left\langle k_{1}, \lambda_{1}, a_{1}\left|\mathcal{T} \varepsilon_{A} \cdot A^{b}\left(x+s \varepsilon_{A}\right): V_{g g g}(y):\right| k_{2}, \lambda_{2}, a_{2}\right\rangle, \\
M_{2}= & \frac{i^{2} g}{\pi} \operatorname{Tr}\left(t^{c} t^{b} t^{b^{\prime}}\right) \int_{-\infty}^{\infty} d s \int_{-\infty}^{s} d s^{\prime} \\
& \left\langle k_{1}, \lambda_{1}, a_{1}\left|\mathcal{T} \varepsilon_{A} \cdot A^{b}\left(x+s \varepsilon_{A}\right) \varepsilon_{A} \cdot A^{b^{\prime}}\left(x+s^{\prime} \varepsilon_{A}\right)\right| k_{2}, \lambda_{2}, a_{2}\right\rangle .
\end{aligned}
$$

Above, $\mathcal{T}$ denotes the time ordering of fields while the colon the normal ordering of fields.

Let us first calculate $M_{1}$. Using the Wick theorem we get

$$
\begin{aligned}
M_{1}= & -\frac{i g}{2 \pi} f_{a c d} \delta_{c_{A} b} \varepsilon_{A}^{\alpha} \int_{-\infty}^{\infty} d s \int \widehat{d q} \int d^{4} y d^{4} w q^{\beta} e^{-i(y-w) \cdot q} \\
& \left\{S_{\alpha \nu}^{a b}\left(x-w+s \varepsilon_{A}\right)\left\langle k_{1}, \lambda_{1}, a_{1}\left|: A_{\beta}^{c}(y) A^{d \nu}(y):\right| k_{2}, \lambda_{2}, a_{2}\right\rangle\right. \\
& +S_{\alpha \beta}^{b c}\left(x-y+s \varepsilon_{A}\right)\left\langle k_{1}, \lambda_{1}, a_{1}\left|: A_{\nu}^{a}(w) A^{d \nu}(y):\right| k_{2}, \lambda_{2}, a_{2}\right\rangle \\
& \left.+S_{\alpha \nu}^{b d}\left(x-y+s \varepsilon_{A}\right)\left\langle k_{1}, \lambda_{1}, a_{1}\left|: A^{a \nu}(w) A_{\beta}^{c}(y):\right| k_{2}, \lambda_{2}, a_{2}\right\rangle\right\},
\end{aligned}
$$

where

$$
S_{\mu \nu}^{a b}(x)=\int \widehat{d p} e^{-i p \cdot x} \frac{-i \eta_{\mu \nu}}{p^{2}+i \epsilon}
$$

is a position space Feynman propagator for a gluon. For the subsequent matrix elements we get explicitly

$$
\begin{aligned}
\left\langle k_{1}, \lambda_{1}, c_{1}\left|: A_{\beta}^{c}(y) A^{d \nu}(y):\right| k_{2}, \lambda_{2}, c_{2}\right\rangle= & \\
=e^{i y \cdot\left(k_{1}-k_{2}\right)}\left[\delta_{c c_{1}} \delta_{d c_{2}} \varepsilon_{\beta}^{\lambda_{1} *}\left(k_{1}\right) \varepsilon^{\lambda_{2} \nu}\left(k_{2}\right)\right. & \left.+\delta_{d c_{1}} \delta_{c c_{2}} \varepsilon_{\beta}^{\lambda_{2}}\left(k_{2}\right) \varepsilon^{\lambda_{1} \nu *}\left(k_{1}\right)\right], \\
\left\langle k_{1}, \lambda_{1}, c_{1}\left|: A_{\nu}^{a}(w) A^{d \nu}(y):\right| k_{2}, \lambda_{2}, c_{2}\right\rangle= & \delta_{a c_{1}} \delta_{d c_{2}} \varepsilon_{\nu}^{\lambda_{1} *}\left(k_{1}\right) \varepsilon^{\lambda_{2} \nu}\left(k_{2}\right) e^{i w \cdot k_{1}-i y \cdot k_{2}} \\
& +\delta_{d c_{1}} \delta_{a c_{2}} \varepsilon_{\nu}^{\lambda_{2}}\left(k_{2}\right) \varepsilon^{\lambda_{1} \nu *}\left(k_{1}\right) e^{-i w \cdot k_{2}+i y \cdot k_{1}} \\
\left\langle k_{1}, \lambda_{1}, c_{1}\left|: A^{a \nu}(w) A_{\beta}^{c}(y):\right| k_{2}, \lambda_{2}, c_{2}\right\rangle= & \delta_{a c_{1}} \delta_{c c_{2}} \varepsilon^{\lambda_{1} \nu *}\left(k_{1}\right) \varepsilon_{\beta}^{\lambda_{2}}\left(k_{2}\right) e^{i w \cdot k_{1}-i y \cdot k_{2}} \\
& +\delta_{c c_{1}} \delta_{a c_{2}} \varepsilon^{\lambda_{2} \nu}\left(k_{2}\right) \varepsilon_{\beta}^{\lambda_{1} *}\left(k_{1}\right) e^{-i w \cdot k_{2}+i y \cdot k_{1}} .
\end{aligned}
$$


Inserting this to $M_{1}$ we get

$$
\begin{aligned}
M_{1}=- & \frac{i g}{2 \pi} f_{a c d} \delta_{c_{A} b} \varepsilon_{A}^{\alpha} \int_{-\infty}^{\infty} d s \int d^{4} q d^{4} p q^{\beta} e^{-i p \cdot x} e^{-i s p \cdot \varepsilon_{A}} \frac{-i}{p^{2}} \\
& \left\{\eta_{\alpha \nu} \delta^{4}(q+p) \delta^{4}\left(q-k_{1}+k_{2}\right) \delta_{a b}\right. \\
& {\left[\delta_{c c_{1}} \delta_{d c_{2}} \varepsilon_{\beta}^{\lambda_{1} *}\left(k_{1}\right) \varepsilon^{\lambda_{2} \nu}\left(k_{2}\right)+\delta_{d c_{1}} \delta_{c c_{2}} \varepsilon_{\beta}^{\lambda_{2}}\left(k_{2}\right) \varepsilon^{\lambda_{1} \nu *}\left(k_{1}\right)\right] } \\
& +\eta_{\alpha \beta}\left[\delta\left(q+k_{1}\right) \delta\left(q-p+k_{2}\right) \delta_{b c} \delta_{a c_{1}} \delta_{d c_{2}} \varepsilon_{\nu}^{\lambda_{1} *}\left(k_{1}\right) \varepsilon^{\lambda_{2} \nu}\left(k_{2}\right)\right. \\
& \left.+\delta\left(q-k_{2}\right) \delta\left(q-p-k_{1}\right) \delta_{b c} \delta_{d c_{1}} \delta_{a c_{2}} \varepsilon_{\nu}^{\lambda_{2}}\left(k_{2}\right) \varepsilon^{\lambda_{1} \nu *}\left(k_{1}\right)\right] \\
& +\eta_{\alpha \nu}\left[\delta\left(q+k_{1}\right) \delta\left(q-p+k_{2}\right) \delta_{b d} \delta_{a c_{1}} \delta_{c c_{2}} \varepsilon^{\lambda_{1} \nu *}\left(k_{1}\right) \varepsilon_{\beta}^{\lambda_{2}}\left(k_{2}\right)\right. \\
& \left.\left.+\delta\left(q-k_{2}\right) \delta\left(q-p-k_{1}\right) \delta_{b d} \delta_{c c_{1}} \delta_{a c_{2}} \varepsilon^{\lambda_{2} \nu}\left(k_{2}\right) \varepsilon_{\beta}^{\lambda_{1} *}\left(k_{1}\right)\right]\right\} .
\end{aligned}
$$

Now, we perform the Fourier transform with respect to $x$ and utilize the deltas

$$
\begin{aligned}
\tilde{M}_{1}= & -\frac{g}{2 \pi} \delta^{4}\left(k_{A}+k_{1}-k_{2}\right) \int_{-\infty}^{\infty} d s e^{-i s k_{A} \cdot \varepsilon_{A}} \frac{\varepsilon_{A}^{\alpha}}{k_{A}^{2}} f_{c_{A} c_{1} c_{2}} \\
& \left\{-\eta_{\alpha \nu} k_{A}^{\beta}\left[\varepsilon_{\beta}^{\lambda_{1} *}\left(k_{1}\right) \varepsilon^{\lambda_{2} \nu}\left(k_{2}\right)-\varepsilon_{\beta}^{\lambda_{2}}\left(k_{2}\right) \varepsilon^{\lambda_{1} \nu *}\left(k_{1}\right)\right]\right. \\
& +\eta_{\alpha \beta}\left[k_{1}^{\beta} \varepsilon_{\nu}^{\lambda_{1} *}\left(k_{1}\right) \varepsilon^{\lambda_{2} \nu}\left(k_{2}\right)+k_{2}^{\beta} \varepsilon_{\nu}^{\lambda_{2}}\left(k_{2}\right) \varepsilon^{\lambda_{1} \nu *}\left(k_{1}\right)\right] \\
& \left.-\eta_{\alpha \nu}\left[k_{1}^{\beta} \varepsilon^{\lambda_{1} \nu *}\left(k_{1}\right) \varepsilon_{\beta}^{\lambda_{2}}\left(k_{2}\right)+k_{2}^{\beta} \varepsilon^{\lambda_{2} \nu}\left(k_{2}\right) \varepsilon_{\beta}^{\lambda_{1} *}\left(k_{1}\right)\right]\right\} .
\end{aligned}
$$

Using

$$
\int_{-\infty}^{\infty} d s e^{-i s p \cdot \varepsilon_{A}}=2 \pi \delta\left(p \cdot \varepsilon_{A}\right)
$$

and rearranging the terms we get

$$
\begin{aligned}
\tilde{M}_{1}= & -g \delta^{4}\left(k_{A}+k_{1}-k_{2}\right) \delta\left(k_{A} \cdot \varepsilon_{A}\right) \frac{\varepsilon_{A}^{\alpha} \eta_{\alpha \gamma}}{k_{A}^{2}} f_{c_{A} c_{1} c_{2}} \\
& {\left[\eta^{\alpha_{1} \alpha_{2}}\left(k_{1}^{\gamma}+k_{2}^{\gamma}\right)+\eta^{\gamma \alpha_{1}}\left(k_{A}^{\alpha_{2}}-k_{1}^{\alpha_{2}}\right)+\eta^{\alpha_{2} \gamma}\left(-k_{2}^{\alpha_{1}}-k_{A}^{\alpha_{1}}\right)\right] \varepsilon_{\alpha_{1}}^{\lambda_{1} *}\left(k_{1}\right) \varepsilon_{\alpha_{2}}^{\lambda_{2}}\left(k_{2}\right) . }
\end{aligned}
$$

Let us note, that in the square bracket we have the standard triple-gluon vertex.

Next let us calculate $M_{2}$. We have

$$
\begin{aligned}
M_{2}= & -\frac{g}{\pi} \operatorname{Tr}\left(t^{c_{A}} t^{b} t^{b^{\prime}}\right) \varepsilon_{A}^{\alpha} \varepsilon_{A}^{\beta} \int_{-\infty}^{\infty} d s \int_{-\infty}^{s} d s^{\prime} \\
& \left\langle k_{1}, \lambda_{1}, a_{1}\left|: A_{\alpha}^{b}\left(x+s \varepsilon_{A}\right) A_{\beta}^{b^{\prime}}\left(x+s^{\prime} \varepsilon_{A}\right):\right| k_{2}, \lambda_{2}, a_{2}\right\rangle .
\end{aligned}
$$

Further

$$
\begin{aligned}
& \left\langle k_{1}, \lambda_{1}, a_{1}\left|: A_{\alpha}^{b}\left(x+s \varepsilon_{A}\right) A_{\beta}^{b^{\prime}}\left(x+s^{\prime} \varepsilon_{A}\right):\right| k_{2}, \lambda_{2}, a_{2}\right\rangle= \\
& =e^{i x \cdot\left(k_{1}-k_{2}\right)}\left[e^{i s \varepsilon_{A} \cdot k_{1}} e^{-i s^{\prime} \varepsilon_{A} \cdot k_{2}} \delta_{b c_{1}} \delta_{b^{\prime} c_{2}} \varepsilon_{\alpha}^{\lambda_{1} *}\left(k_{1}\right) \varepsilon_{\beta}^{\lambda_{2}}\left(k_{2}\right)\right. \\
& \left.\quad+e^{-i s \varepsilon_{A} \cdot k_{2}} e^{i s^{\prime} \varepsilon_{A} \cdot k_{1}} \delta_{b c_{2}} \delta_{b^{\prime} c_{1}} \varepsilon_{\alpha}^{\lambda_{2}}\left(k_{2}\right) \varepsilon_{\beta}^{\lambda_{1} *}\left(k_{1}\right)\right] .
\end{aligned}
$$


Thus we have

$$
\begin{aligned}
M_{2}= & -\frac{g}{\pi} \operatorname{Tr}\left(t^{c} t^{b} t^{b^{\prime}}\right) \varepsilon_{A}^{\alpha} \varepsilon_{A}^{\beta} \int_{-\infty}^{\infty} d s \int_{-\infty}^{s} d s^{\prime} e^{i x \cdot\left(k_{1}-k_{2}\right)} \\
& {\left[e^{i s \varepsilon_{A} \cdot k_{1}} e^{-i s^{\prime} \varepsilon_{A} \cdot k_{2}} \delta_{b c_{1}} \delta_{b^{\prime} c_{2}} \varepsilon_{\alpha}^{\lambda_{1} *}\left(k_{1}\right) \varepsilon_{\beta}^{\lambda_{2}}\left(k_{2}\right)\right.} \\
& \left.+e^{-i s \varepsilon_{A} \cdot k_{2}} e^{i s^{\prime} \varepsilon_{A} \cdot k_{1}} \delta_{b c_{2}} \delta_{b^{\prime} c_{1}} \varepsilon_{\alpha}^{\lambda_{2}}\left(k_{2}\right) \varepsilon_{\beta}^{\lambda_{1} *}\left(k_{1}\right)\right]
\end{aligned}
$$

Next, we have to integrate over the path parameters. Using the $i \epsilon$ prescription (4.15) we have

$$
\begin{aligned}
\int_{-\infty}^{\infty} d s \int_{-\infty}^{s} d s^{\prime} e^{i s \varepsilon_{A} \cdot k_{1}} e^{-i s^{\prime} \varepsilon_{A} \cdot k_{2}} & =\frac{2 \pi i}{\varepsilon_{A} \cdot k_{2}+i \epsilon} \delta\left(\varepsilon_{A} \cdot k_{1}-\varepsilon_{A} \cdot k_{2}\right), \\
\int_{-\infty}^{\infty} d s \int_{-\infty}^{s} d s^{\prime} e^{-i s \varepsilon_{A} \cdot k_{2}} e^{i s^{\prime} \varepsilon_{A} \cdot k_{1}} & =\frac{2 \pi i}{-\varepsilon_{A} \cdot k_{1}+i \epsilon} \delta\left(\varepsilon_{A} \cdot k_{1}-\varepsilon_{A} \cdot k_{2}\right) .
\end{aligned}
$$

Taking the Fourier transform we get

$$
\begin{gathered}
\tilde{M}_{2}=-2 g \delta^{4}\left(k_{A}+k_{1}-k_{2}\right) \varepsilon_{A} \cdot \varepsilon^{\lambda_{1} *}\left(k_{1}\right) \varepsilon_{A} \cdot \varepsilon^{\lambda_{2}}\left(k_{2}\right) \\
\delta\left(\varepsilon_{A} \cdot k_{A}\right)\left[\operatorname{Tr}\left(t^{c_{A}} t^{c_{1}} t^{c_{2}}\right) \frac{i}{\varepsilon_{A} \cdot k_{2}}\right. \\
\left.+\operatorname{Tr}\left(t^{c_{A}} t^{c_{2}} t^{c_{1}}\right) \frac{-i}{\varepsilon_{A} \cdot k_{1}}\right]
\end{gathered}
$$

or

$$
\tilde{M}_{2}=g \delta^{4}\left(k_{A}+k_{1}-k_{2}\right) \varepsilon_{A} \cdot \varepsilon^{\lambda_{1} *}\left(k_{1}\right) \varepsilon_{A} \cdot \varepsilon^{\lambda_{2}}\left(k_{2}\right) \frac{1}{\varepsilon_{A} \cdot k_{1}} f_{c_{A} c_{1} c_{2}} .
$$

Collecting $\tilde{M}_{1}$ and $\tilde{M}_{2}$ we get

$$
\begin{aligned}
\mathfrak{M}\left(\varepsilon_{A} ; \varepsilon_{1}, \varepsilon_{2}\right)= & -g \delta^{4}\left(k_{A}+k_{1}-k_{2}\right) \delta\left(k_{A} \cdot \varepsilon_{A}\right) f_{c_{A} c_{1} c_{2}} \varepsilon_{A \gamma} \varepsilon_{\alpha_{1}}^{\lambda_{1} *}\left(k_{1}\right) \varepsilon_{\alpha_{2}}^{\lambda_{2}}\left(k_{2}\right) \\
& \left\{\frac{1}{k_{A}^{2}}\left[\eta^{\alpha_{1} \alpha_{2}}\left(k_{1}^{\gamma}+k_{2}^{\gamma}\right)+\eta^{\alpha_{1}}\left(k_{A}^{\alpha_{2}}-k_{1}^{\alpha_{2}}\right)+\eta^{\alpha_{2} \gamma}\left(-k_{2}^{\alpha_{1}}-k_{A}^{\alpha_{1}}\right)\right]\right. \\
& \left.\quad-\frac{\varepsilon_{A}^{\alpha_{1}} \varepsilon_{A}^{\alpha_{2}}}{\varepsilon_{A} \cdot k_{1}}\right\}
\end{aligned}
$$

Let us note, that for $\varepsilon_{A}$ taken to be $n_{+}$or $n_{-}$and after multiplying by $k_{A}^{2}$ it coincides with the RPP vertex of [12].

\section{B The BRST invariance of the $\mathcal{R}$ operator}

In this appendix we argue that the state (4.27) defined by the action of the operator (4.26) on the vacuum state belongs to the cohomology of the BRST transformation. For a pedagogical review of the subject we refer to [35]. Here, let us just recall the necessary basic facts. Let $Q_{\mathrm{BRST}}$ be a charge generating the BRST transformation. It is nilpotent, i.e. $Q_{\mathrm{BRST}}^{2}=0$. Below, the following commutation relation will be useful: $i\left[Q_{\mathrm{BRST}}, A_{\mu}^{a}\right]=D_{\mu}^{a b} c^{b}$, where $D_{\mu}^{a b}=\delta^{a b} \partial_{\mu}-g f^{a b c} A_{\mu}^{c}$ and $c^{b}$ is the ghost field. A state $|\phi\rangle$ 
belongs to the cohomology of $Q_{\mathrm{BRST}}$ if it belongs to the kernel of $Q_{\mathrm{BRST}}$ (i.e. $Q_{\mathrm{BRST}}|\phi\rangle=0$ ) but not to the image. From nilpotency it directly follows that any state in the image has zero norm. It is the basic requirement for physical states that they belong to the cohomology of the BRST transformation.

Let us now turn to the $\mathcal{R}_{n}^{c}$ operator. Note that since the vacuum state belongs to the cohomology and since the state $\mathcal{R}_{n}^{c}|0\rangle$ has a nonzero norm, it is enough to show that

$$
\left[\mathcal{R}_{n}^{d}(k), Q_{\mathrm{BRST}}\right]=0 .
$$

To this end we expand the definition (4.26) of $\mathcal{R}_{n}^{c}$. The first nontrivial term of the expansion of (B.1) is

$$
\begin{aligned}
& i \int \frac{d k^{(n)}}{2 \pi} \int d^{4} x e^{i x \cdot k} \int_{-\infty}^{\infty} d s n^{\mu} t^{a}\left[Q_{\mathrm{BRST}}, A_{\mu}^{a}(x+s n)\right] \\
& =-i \int d k^{(n)} \delta(k \cdot n) k \cdot n t^{a} \tilde{c}^{a}(k) \\
& \quad-g \int d k^{(n)} \int \frac{d^{4} p}{(2 \pi)^{4}} \delta(k \cdot n) t^{a} f^{a b c} n^{\mu} \tilde{A}_{\mu}^{c}(p) \tilde{c}^{b}(k-p),
\end{aligned}
$$

where we have switched to the Fourier space (the tildes denote Fourier-transformed fields). Note that the first term on the r.h.s. vanishes after integrating the delta function. The second term of the expansion of the commutator (B.1) is

$$
\begin{aligned}
i^{2} g \int \frac{d k^{(n)}}{2 \pi} \int d^{4} x e^{i x \cdot k} \int_{-\infty}^{\infty} d s \int_{-\infty}^{s} d s^{\prime} n^{\mu} n^{\nu} t^{a} t^{a^{\prime}}\left[Q_{\mathrm{BRST}}, A_{\mu}^{a}(x+s n) A_{\nu}^{a^{\prime}}\left(x+s^{\prime} n\right)\right] \\
=g \int d k^{(n)} \int \frac{d^{4} p}{(2 \pi)^{4}} \frac{d^{4} p^{\prime}}{(2 \pi)^{4}} \delta^{4}\left(k-p-p^{\prime}\right) \delta\left(p \cdot n+p^{\prime} \cdot n\right) p^{\prime} \cdot n \\
\quad\left\{\frac{i}{p^{\prime} \cdot n+i \epsilon} t^{a} t^{a^{\prime}}+\frac{i}{p \cdot n+i \epsilon} t^{a^{\prime}} t^{a}\right\} n^{\mu} \tilde{A}_{\mu}^{a}(p) c^{a^{\prime}}\left(p^{\prime}\right)+\mathcal{O}\left(g^{2}\right) \\
=i g \int d k^{(n)} \int \frac{d^{4} p}{(2 \pi)^{4}} \delta(k \cdot n)\left[t^{a}, t^{a^{\prime}}\right] n^{\mu} \tilde{A}_{\mu}^{a}(p) \tilde{c}^{a^{\prime}}(k-p)+\mathcal{O}\left(g^{2}\right),
\end{aligned}
$$

where we have suppressed the term of the order of $g^{2}$. Using $\left[t^{a}, t^{a^{\prime}}\right]=i f^{a a^{\prime} b} t^{b}$ and reshuffling the indices we see that the first term on the r.h.s. cancels against (B.2). Similar canceling will occur order by order in $g$.

Open Access. This article is distributed under the terms of the Creative Commons Attribution License (CC-BY 4.0), which permits any use, distribution and reproduction in any medium, provided the original author(s) and source are credited.

\section{References}

[1] J. Collins, Cambridge Monographs on Particle Physics, Nuclear Physics and Cosmology. Vol. 12: Foundations of perturbative QCD, Cambridge University Press, Cambridge U.K. (2011). 
[2] L.V. Gribov, E.M. Levin and M.G. Ryskin, Semihard Processes in QCD, Phys. Rept. 100 (1983) 1 [INSPIRE].

[3] S. Catani, M. Ciafaloni and F. Hautmann, High-energy factorization and small $x$ heavy flavor production, Nucl. Phys. B 366 (1991) 135 [InSPIRE].

[4] J.C. Collins and R.K. Ellis, Heavy quark production in very high-energy hadron collisions, Nucl. Phys. B 360 (1991) 3 [INSPIRE].

[5] S. Catani and F. Hautmann, High-energy factorization and small $x$ deep inelastic scattering beyond leading order, Nucl. Phys. B 427 (1994) 475 [hep-ph/9405388] [InSPIRE].

[6] B.-W. Xiao and F. Yuan, Non-Universality of Transverse Momentum Dependent Parton Distributions at Small-x, Phys. Rev. Lett. 105 (2010) 062001 [arXiv:1003.0482] [INSPIRE].

[7] F. Dominguez, C. Marquet, B.-W. Xiao and F. Yuan, Universality of Unintegrated Gluon Distributions at small x, Phys. Rev. D 83 (2011) 105005 [arXiv:1101.0715] [INSPIRE].

[8] P.J. Mulders and T.C. Rogers, Gauge Links, TMD-Factorization and TMD-Factorization Breaking, arXiv:1102.4569 [INSPIRE].

[9] E. Avsar, On the Understanding and Use of 'Unintegrated' Parton Distributions in Small-x QCD, Int. J. Mod. Phys. Conf. Ser. 04 (2011) 74 [arXiv:1108.1181] [INSPIRE].

[10] E. Avsar, TMD factorization and the gluon distribution in high energy $Q C D$, arXiv: 1203.1916 [INSPIRE].

[11] L.N. Lipatov, Gauge invariant effective action for high-energy processes in QCD, Nucl. Phys. B 452 (1995) 369 [hep-ph/9502308] [INSPIRE].

[12] E.N. Antonov, L.N. Lipatov, E.A. Kuraev and I.O. Cherednikov, Feynman rules for effective Regge action, Nucl. Phys. B 721 (2005) 111 [hep-ph/0411185] [INSPIRE].

[13] A. van Hameren, P. Kotko and K. Kutak, Helicity amplitudes for high-energy scattering, JHEP 01 (2013) 078 [arXiv: 1211.0961] [INSPIRE].

[14] A. van Hameren, P. Kotko and K. Kutak, Multi-gluon helicity amplitudes with one off-shell leg within high energy factorization, JHEP 12 (2012) 029 [arXiv:1207.3332] [INSPIRE].

[15] A. van Hameren, K. Kutak and T. Salwa, Scattering amplitudes with off-shell quarks, Phys. Lett. B 727 (2013) 226 [arXiv:1308.2861] [InSPIRE].

[16] A. van Hameren, P. Kotko and K. Kutak, Three jet production and gluon saturation effects in $p$ - $p$ and $p$ - $P b$ collisions within high-energy factorization, Phys. Rev. D 88 (2013) 094001 [arXiv: 1308.0452] [INSPIRE].

[17] A. van Hameren, P. Kotko, K. Kutak, C. Marquet and S. Sapeta, Saturation effects in forward-forward dijet production in p+Pb collisions, Phys. Rev. D 89 (2014) 094014 [arXiv: 1402.5065] [INSPIRE].

[18] A. Leonidov and D. Ostrovsky, Angular and momentum asymmetry in particle production at high-energies, Phys. Rev. D 62 (2000) 094009 [hep-ph/9905496] [INSPIRE].

[19] I. Balitsky, High-energy QCD and Wilson lines, hep-ph/0101042 [INSPIRE].

[20] F. Gelis, E. Iancu, J. Jalilian-Marian and R. Venugopalan, The Color Glass Condensate, Ann. Rev. Nucl. Part. Sci. 60 (2010) 463 [arXiv: 1002.0333] [InSPIRE].

[21] J. Kuipers, T. Ueda, J.A.M. Vermaseren and J. Vollinga, FORM version 4.0, Comput. Phys. Commun. 184 (2013) 1453 [arXiv:1203.6543] [INSPIRE]. 
[22] M. Deak, F. Hautmann, H. Jung and K. Kutak, Forward Jet Production at the Large Hadron Collider, JHEP 09 (2009) 121 [arXiv:0908.0538] [INSPIRE].

[23] H. Arodz and L. Hadasz, Lectures on Classical and Quantum Theory of Fields, Springer, Berlin Germany (2010).

[24] M.L. Mangano, S.J. Parke and Z. Xu, Duality and Multi-Gluon Scattering, Nucl. Phys. B 298 (1988) 653 [INSPIRE].

[25] M.L. Mangano and S.J. Parke, Multiparton amplitudes in gauge theories, Phys. Rept. 200 (1991) 301 [hep-th/0509223] [INSPIRE].

[26] J.C. Collins and D.E. Soper, Parton Distribution and Decay Functions, Nucl. Phys. B 194 (1982) 445 [INSPIRE].

[27] P. Kotko, OGIME - Off-shell Gauge Invariant Matrix Elements (2013), FORM program, available at http://annapurna.ifj.edu.pl/ pkotko/OGIME.html.

[28] J. Bartels, L.N. Lipatov and G.P. Vacca, Ward Identities for Amplitudes with Reggeized Gluons, Phys. Rev. D 86 (2012) 105045 [arXiv: 1205.2530] [InSPIRE].

[29] M.A. Braun and M.I. Vyazovsky, The reggeon $\rightarrow 2$ reggeons + particle vertex in the Lipatov effective action formalism, Eur. Phys. J. C 51 (2007) 103 [hep-ph/0612323] [INSPIRE].

[30] M.A. Braun, M.Y. Salykin, S.S. Pozdnyakov and M.I. Vyazovsky, Production of a gluon with the exchange of three reggeized gluons in the Lipatov effective action approach, Eur. Phys. J. C 72 (2012) 2223 [arXiv:1209.2490] [INSPIRE].

[31] E. Richter-Was and Z. Was, Orbital angular momentum in the QCD evolution, Tech. Rep. CPT-2080. M-CPT-2080, CNRS Marseille, Cent. Phys. Theor., Marseille France (1988).

[32] I. Balitsky, Operator expansion for high-energy scattering, Nucl. Phys. B 463 (1996) 99 [hep-ph/9509348] [INSPIRE].

[33] S. Caron-Huot, When does the gluon reggeize?, arXiv:1309.6521 [INSPIRE].

[34] D. Binosi and L. Theussl, JaxoDraw: a graphical user interface for drawing Feynman diagrams, Comput. Phys. Commun. 161 (2004) 76 [hep-ph/0309015] [INSPIRE].

[35] S. Weinberg, The quantum theory of fields. Vol. 2: Modern applications, Cambridge University Press, Cambridge U.K. (2005). 